11 Department of Cellular and Molecular Medicine, University of Arizona

$12{ }^{2}$ University of Arizona Cancer Center

$13{ }^{3}$ Department of Cancer Biology, University of Arizona

$14{ }^{4}$ Department of Microbiology, Immunology \& Cancer Biology, University of Virginia

15

16 Running title: PIM controls mitochondrial dynamics and chemosensitivity

\section{PIM Kinases Alter Mitochondrial Dynamics and Chemosensitivity in Lung Cancer}

Shailender S. Chauhan ${ }^{1}$, Rachel K. Toth², Corbin C. Jensen ${ }^{3}$, Andrea L. Casillas ${ }^{3}$, David F. Kashatus ${ }^{4}$, Noel A. Warfel ${ }^{1,2^{*}}$ 
35 Abstract: Resistance to chemotherapy represents a major obstacle to the successful

36 treatment of non-small cell lung cancer (NSCLC). The goal of this study was to determine

37 how PIM kinases impact mitochondrial dynamics, ROS production, and response to

38 chemotherapy in lung cancer. Live cell imaging and microscopy were used to determine

39 the effect of PIM loss or inhibition on mitochondrial phenotype and ROS. Inhibition of PIM

40 kinases caused excessive mitochondrial fission and significant upregulation of

41 mitochondrial superoxide, increasing intercellular ROS. Mechanistically, we define a

42 signaling axis linking PIM1 to Drp1 and mitochondrial fission in lung cancer. PIM inhibition

43 significantly increased the protein levels and mitochondrial localization of Drp1, causing

44 marked fragmentation of mitochondria. An inverse correlation between PIM1 and Drp1

45 was confirmed in NSCLC patient samples. Inhibition of PIM sensitized NSCLC to

46 chemotherapy and produced a synergistic anti-tumor response in vitro and in vivo.

47 Immunohistochemistry and transmission electron microscopy verified that PIM inhibitors

48 promote mitochondrial fission and apoptosis in vivo. These data improve our knowledge

49 about how PIM1 regulates mitochondria and provide justification for combining PIM

50 inhibition with chemotherapy in NSCLC.

52 Keywords: PIM kinases, Drp1, mitochondrial dynamics, chemotherapy, reactive oxygen

53 species 
56 Introduction: Lung cancer is the second most commonly diagnosed type of cancer and

57 the leading cause of cancer-related mortality worldwide. More than two-thirds of lung 58 cancer patients are diagnosed at an advance stage (III-IV), and intrinsic and/or acquired

59 resistance to treatment represent major obstacles to the successful treatment of patients

60 with advanced disease $(1,2)$. As compared to other types of lung cancer, non-small cell

61 lung carcinomas (NSCLC) is less prone to undergo spontaneous and treatment-induced

62 apoptosis (3), suggesting that deficiencies in the apoptotic process may be responsible

63 for their and/or acquired resistance to chemotherapy (4).

64 Cumulative evidence has demonstrated that an imbalance of mitochondrial fission

65 and fusion is common in cancer (5). Mitochondria exist as a dynamic network that is 66 constantly undergoing fusion (elongation) and fission (fragmentation). Mitochondrial

67 fusion results in a tubular mitochondrial network that serves to counteract metabolic 68 insults, maintain cellular integrity, and provide protection against cell death $(6,7)$. In 69 contrast, mitochondrial fission creates small and fragmented mitochondria, which can

70 have both pro- and anti-tumor effects depending on the cellular context (8). Mitochondrial

71 fission is required for cell division and has been shown to positively regulate cell

72 proliferation in cancer cells (9). However, in response to apoptotic stimuli and cellular

73 stress, too much fission generates excessive reactive oxygen species (ROS) and is a 74 necessary event for the initiation of apoptosis (10). Mitochondrial fusion is associated with 75 chemoresistance in several cancer types, including lung cancer $(7,11)$. Moreover, 76 excessive mitochondrial fragmentation is associated with chemosensitive cancer cells in

77 response to platinum-based therapy, whereas chemoresistant cancer cells retain a more

78 fused mitochondrial network (12). These data demonstrate that mitochondrial fusion is an

79 important event in the acquisition of chemoresistance.

80 An essential step for mitochondrial membrane fission is the recruitment of 81 dynamin-related protein 1 (Drp1) to mitochondria. Drp1 is recruited from the cytoplasm to 
82 the mitochondrial outer membrane $(13,14)$, where it colocalizes with receptors (15). Drp1

83 protein oligomerize to form a ring around the mitochondria that constricts and separates

84 the mitochondrial membrane. Previous reports indicate that downregulation of Drp1 limits

85 mitochondrial fragmentation, cytochrome c release, caspase activation and apoptosis

86 (16), and reduced levels of Drp1 have been reported in many cancers, including NSCLC.

87 Adenocarcinomic alveolar epithelial cells, a model of NSCLC, display decreased Drp1

88 protein expression, which promotes elongation of mitochondrial phenotypes and limits

89 fission while inhibiting the downstream processes of apoptotic activation (17). These

90 observations suggest that enhancing mitochondrial fission is a promising approach to

91 inducing apoptosis in lung cancer (18).

92 The Proviral Integration site for Moloney murine leukemia virus (PIM) kinases are

93 crucial regulators of cell survival and proliferation, and their expression is associated with

94 poor prognosis in several types of cancer (19). A majority of the research on PIM1 has

95 focused on cancers of hematopoietic, prostate or breast origin, and the mechanisms by

96 which PIM1 promotes therapeutic resistance in lung cancer are poorly understood. Our

97 recent work demonstrated that PIM inhibitors cause a marked increase in reactive oxygen

98 species (ROS), which is crucial for their cytotoxic effects toward cancer cells (20).

99 However, the mechanism by which PIM inhibition produces excessive ROS is not well

100 understood. Here, we investigate the consequence of altered PIM kinase expression or

101 activity on mitochondrial dynamics, ROS and therapeutic resistance in lung cancer. 


\section{Results:}

105 High PIM1 is predictive of poor clinical outcome in NSCLC: To study the clinical

106 significance of PIM1 expression in lung cancer, patient samples and publicly available

107 TCGA datasets of human lung cancer cases were analyzed. Immunohistochemical

108 staining of an NSCLC tissue microarray (TMA) comprising normal lung tissue and patient

109 tumors ranging from stage I - III ( $\mathrm{n}=100$ cores) revealed that PIM1 levels were

110 significantly higher levels in all lung cancer cases relative to normal tissue. Patients with

111 stage II and III had significantly higher PIM1 expression than those with stage I (Fig 1A),

112 and the average PIM1 expression in stage III was two-fold higher than that in stage II (Fig

113 1B). Moreover, lung cancer patients with high PIM1 expression had significantly worse

114 survival than those with low PIM1 expression. The median survival time of lung

115 adenocarcinoma patients with high PIM1 expression was significantly shorter than that of

116 patients with low expression at each stage [stage I: 111 vs. 68 mo, stage II: 66 vs. 21 mo,

117 and stage III: 34 vs. 23 mo] (Fig 1C). Notably, patients with high PIM1 displayed

118 significantly worse response to chemotherapy than patients with low PIM1 [stage I: 40 vs.

$1195 \mathrm{mo}$, and stage II: 16 vs. $5 \mathrm{mo}$ (Fig 1D). These, findings suggest that PIM1 upregulation

120 is involved in lung cancer pathogenesis and is significantly associated with resistance to

121 chemotherapy in NSCLC patients.

123 PIM1 inhibition augments mitochondrial superoxide production and ROS

124 accumulation: The fragmented or fused state of mitochondria is critical for maintaining

125 proper function. One of the earliest signs of compromised mitochondria is amplified

126 superoxide production, which ultimately leads to increased production of ROS. Because

127 PIM inhibitors cause a dramatic increase in ROS, we hypothesized that PIM inhibition

128 could generate excess ROS by impairing mitochondrial function. To test this, MitoSOX

129 was used to selectively measure superoxide levels at the mitochondria in WT and Triple 
130 knockout MEFs (TKO; lacking all 3 PIM isoforms), and fold change in corrected total cell

131 fluorescence (CTCF) intensity was measured. TKO MEFs had high basal superoxide

132 levels compared to WT MEFs, and TKO MEFs with PIM1 added back (TKO-PIM1)

133 displayed significantly reduced superoxide (Fig 2A). Similarly, treatment of WT MEFs with

134 a pan-PIM kinase inhibitor (PIM447) caused a 2-fold amplification in superoxide

135 production (Fig 2B). To validate that the observed effects are specific to inhibition of PIM

136 and not an artifact of the drug itself, we treated a panel of NSCLC cell lines (H1299, A549,

137 and $\mathrm{H} 460$ ) with a chemically distinct pan-PIM kinase inhibitor (AZD1208). A similar

138 induction in superoxide production was observed in response to AZD1208 in all cell lines

139 tested, indicating that these effects are specific to PIM inhibition (Fig 2C). Moreover, live

140 cell imaging of mitoSOX demonstrated that superoxide levels were induced within $2 \mathrm{~h}$ of

141 treatment with PIM inhibitors, indicating that blocking PIM has a direct effect on

142 mitochondrial function, independent of its ability to inhibit the Nrf2 antioxidant pathway (Fig

143 2D and Fig S1). To further establish that compromised mitochondrial function following

144 PIM inhibition ultimately causes free radical accumulation, we treated lung cancer cells

145 with AZD1208 for 48 hours and measured ROS levels by EPR spectroscopy. Each of the

146 NSCLC cell lines tested showed a dose-dependent increase in cellular ROS levels in

147 response to PIM inhibition (Fig 2E). These results indicate that PIM1 loss or inhibition

148 impairs mitochondrial function and leads to the accumulation of cellular ROS.

150 PIM1 loss or inhibition induces mitochondrial fragmentation: Based on our finding

151 that small molecule PIM inhibitors increase superoxide as a precursor to intercellular ROS,

152 we speculated that manipulating PIM1 expression and activity could alter mitochondrial

153 dynamics. To test this hypothesis, we assessed the mitochondrial phenotype of WT MEFs,

154 TKO MEFs and TKO-PIM1. Cells were stained with a mitochondrial marker, TOM20, and

155 visualized by fluorescence microscopy. Immunoblotting of IRS1 (S1101), an established 
156 PIM substrate (24), was used as a control to monitor PIM activity. The number of

157 fragmented mitochondria $(<1 \mu \mathrm{m})$ in TKO MEFs was approximately 3-fold higher than that

158 in WT MEFs (Fig 3A-C). Reintroduction of PIM1 to TKO MEFs restored mitochondria to a

159 more elongated state $(>1.5 \mu \mathrm{m})$, similar to that observed in WT MEFs (Fig 3A-C). Next,

160 A549 cells were treated with PIM447 and mitochondrial morphology was quantified by

161 immunofluorescence. Similar to the effect of PIM knockout, small molecule PIM inhibitors

162 significantly increased mitochondrial fragmentation by 3 to 4 -fold compared to control (Fig

163 3D-F). Parallel experiments using AZD1208 or PIM1 overexpression in prostate (Du145),

164 breast (SUM159 and MCF7), and colon cancer (RKO) cell lines showed similar results,

165 indicating that PIM regulates mitochondrial phenotype a broad range of cancers (Fig. S2).

166 The changes in mitochondrial phenotype observed in response to PIM inhibition were

167 further validated using transmission electron microscopy (TEM) in H1299 lung cancer cells

168 treated with AZD1208. Quantification revealed a significant increase in fragmented

169 mitochondria and a decrease in elongated or intermediate (1-1.5 $\mu \mathrm{m})$ mitochondria in

170 response to pharmacological inhibition of PIM (Fig 3G-I and Fig. S3A). To verify that the

171 effects of AZD1208 on mitochondria were independent of Nrf2, a parallel experiment was

172 performed in $\mathrm{H} 1299$ cells with stable knockdown of Keap1, which have constitutive

173 activation of Nrf2. PIM inhibition did not alter Nrf2 levels (Fig S3B) in these cells but

174 significantly increased fragmentation (Fig S3A). Taken together, these findings suggest

175 that PIM controls mitochondrial dynamics in lung cancer.

177 PIM1 loss or inhibition affects mitochondrial phenotype in a Drp1-dependent

178 manner: Drp1 is a key regulator of mitochondrial fission that promotes mitochondrial

179 fragmentation when active. Therefore, we hypothesized that PIM could be controlling Drp1

180 to alter mitochondrial phenotype in lung cancer. To test whether there was an association

181 between PIM1 and Drp1 in human samples, we stained a human lung cancer TMA for 
182 PIM1 and Drp1. Interestingly, areas within tissue cores expressing high levels of PIM1 183 correlated with low Drp1 expression and vice-versa (Fig 4A), and the inverse relationship 184 between these proteins was further exaggerated during disease progression, as 185 determined by assessing the PIM1/Drp1ratio by disease stage (Fig 4B). To verify the 186 inverse correlation between these proteins, we immunoblotted for Drp1 in WT and TKO 187 MEFs, as well as a panel of lung cancer cell lines (H1299, A549, and H460) treated with

188 DMSO or PIM447. Both genetic depletion and chemical inhibition of PIM significantly 189 increased total Drp1 levels (Fig 4C). Posttranslational modifications play an important role 190 in maintaining Drp1 activity, particularly phosphorylation. Phosphorylation at S616 is 191 thought to increase Drp1 activity and mitochondrial fragmentation, whereas 192 phosphorylation at S637 opposes Drp1 activation and increases fusion. Thus, we next 193 examined the phosphorylation state of Drp1 in response to PIM loss or inhibition.

194 Strikingly, phosphorylation of S616 was significantly increased and phosphorylation of 195 S637 was significantly reduced in TKO MEFs compared to WT MEFs (Fig. 4C). Similar 196 effects on Drp1 phosphorylation were observed in lung cancer cells (H1299, A549, and $197 \mathrm{H} 460$ ) following treatment with PIM447 (Fig 4C). To confirm that Drp1 is responsible for 198 the mitochondrial fragmentation observed in response to PIM loss or inhibition, TKO MEFs 199 were treated with Mdivi-1, a Drp1 inhibitor, or incubated in HBSS as positive control to 200 decrease fragmentation and induce fusion (25). After $24 \mathrm{~h}$ incubation with Mdivi-1, the 201 basal number of fragmented mitochondria in TKO MEFs was significantly decreased (Fig 202 4D). Next, we tested whether Drp1 is necessary for PIM inhibitors to induce mitochondrial 203 fragmentation. Treatment of A549 cells with PIM447 caused a 4-fold increase in 204 fragmented mitochondria, whereas mitochondria were refractory to PIM inhibition when 205 Drp1 was inactivated (Fig 4E). Because Mdivi-1 has been shown to have off target effects 206 (26), we generated stable knockdown of Drp1 in A549 and H460 cells to verify our results 207 using this inhibitor (Fig. 4F). Genetic suppression of Drp1 resulted in elongated/fused 
208 mitochondria and a significant decrease in fragmented mitochondria compared to the 209 parental cell lines (Fig S4A). Importantly, mitochondria in cells lacking Drp1 were 210 refractory to fragmentation (Fig 4G) and superoxide production (Fig S4B) upon treatment 211 with PIM447. Thus, PIM inhibitors require the activation of Drp1 to induce mitochondrial 212 fission and increase superoxide generation.

213 Activation of Drp1 via phosphorylation is known to regulate its localization to 214 mitochondria, so we reasoned that loss of PIM would increase the localization of DRP1 to 215 mitochondria. To this end, we used confocal microscopy to assess the co-localization of 216 Drp1 and mitochondria in WT and TKO MEFs. MEFs lacking PIM displayed a significant 217 increase in TOM20 and Drp1 co-localization compared to WT MEFs (Fig 4H). Importantly, 218 addback of PIM1 was sufficient to reduce total Drp1 levels as well as its localization to 219 mitochondria, supporting the elongated phenotype observed in these cells (Fig 3B). Taken 220 together, these results confirm that PIM loss or inhibition increases Drp1 levels and 221 promotes its activity by altering the ratio of activating and inhibitory phosphorylation, 222 ultimately increasing the recruitment of Drp1 to mitochondria, where it increases fission.

224 PIM inhibition sensitizes lung cancer cells to chemotherapy by altering 225 mitochondrial phenotype: Elevated PIM1 expression and elongated mitochondrial 226 morphology have been linked to drug resistance, but the association between these 227 factors has not been tested. We proposed that elongated mitochondrial phenotype 228 associated with PIM1 upregulation is essential for PIM1 to promote chemoresistance in 229 lung cancer. To validate this, A549 and H460 lung cancer cell lines were stably transfected 230 with vector control (pCIP) or PIM1 (hPIM1) plasmids and treated with docetaxel for $72 \mathrm{~h}$ 231 (Fig 5A and 5B). Immunoblotting demonstrated that PIM1-overexpressing cells had 232 reduced total Drp1 levels and altered phosphorylation status (increased S637 and 233 decreased S616). Indeed, cells overexpressing PIM1 displayed an elongated 
234 mitochondrial phenotype (Fig 5C) and were significantly resistant to docetaxel ( IC $_{50}$ values

235 for A549 pCIP vs. hPIM1: 2.2 vs. $3.9 \mathrm{nM}$ and H460: 1.7 vs. $3.4 \mathrm{nM})$ and cisplatin $\left(\mathrm{IC}_{50}\right.$

236 values for A549 pCIP vs. hPIM1: 1.9 to $3.3 \mu \mathrm{M}$ and H460: 1.5 vs. $4.4 \mu \mathrm{M})$ (Fig 5D). Since

237 PIM1 levels are significantly higher in advanced lung cancer (Fig 1A), and its

238 overexpression can lead to chemoresistance, we hypothesized that PIM inhibition would

239 sensitize lung cancer cells to chemotherapy. To test this, A549, and H460 cells were

240 treated with AZD1208 and docetaxel for $72 \mathrm{~h}$ and cell viability was measured by crystal

241 violet staining. Dose-response curves and $\mathrm{Cl}$ calculation revealed a synergistic anti-tumor

242 response of combined treatment with AZD1208 and docetaxel (Fig S5A-C). To confirm

243 that mitochondrial fragmentation is required for PIM inhibitors to sensitize lung cancer cells

244 to chemotherapy, we treated shDRP1 cells with AZD1208 and docetaxel. Noticeably less

245 synergy was observed between the PIM inhibitor and docetaxel in cells lacking Drp1,

246 indicating that the ability of PIM inhibitors to cause fragmentation is critical for their ability

247 to sensitize lung cancer cells to chemotherapy (Fig S5D-F). To determine whether

248 combined treatment of PIM and docetaxel places added stress on mitochondria and

249 enhances the accumulation of intercellular ROS, A549 and H460 cells were stained with

250 H2DCF-DA and ROS was measured by flow cytometry. Inhibition of PIM alone amplified

251 ROS in both A549 and H460 cells, and the combination of AZD1208 and docetaxel caused

252 a further significant increase in ROS (Fig 5E).

253 To determine how PIM1 inhibition sensitizes lung cancer cells to docetaxel, we

254 assessed markers related to mitochondrial phenotype and apoptotic cell death.

255 Combination therapy induced the expression of markers of mitochondrial fragmentation

256 and increased activation of the apoptotic pathway (i.e., downregulation of Bcl-2 and

257 increased cleaved-caspase 3 (CC3)) (Fig 5F). To verify that cells treated with the

258 combination were undergoing apoptosis initiated at the mitochondria, we measured the

259 effect of combination therapy on changes in mitochondrial membrane potential (MMP), 
260 which is a precursor to cytochrome-c release and caspase activation, via JC-1 staining 261 assay. A panel of lung cancer cells were first screened for JC-1 aggregates in the 262 presence or absence of $\mathrm{H}_{2} \mathrm{O}_{2}$ to indicate changes in MMP, and a $24 \mathrm{~h}$ time point was 263 chosen for subsequent drug treatment (Fig S5G). Cells treated with the combination of 264 AZD1208 and docetaxel exhibited hyper depolarization of mitochondria that was greater 265 than with either agent alone, indicative of the loss of MMP (Fig $5 \mathrm{G}$ and $5 \mathrm{H}$ ). Combined treatment with PIM inhibitor and docetaxel has synergistic anti-tumor 268 effects in a xenograft model of lung cancer: Based on our findings in vitro, we next 269 investigated the effect of PIM1 inhibition and/or docetaxel treatment on tumor growth, 270 proliferation, and apoptosis in vivo. Five million A549 cells were injected subcutaneously 271 into each flank of SCID mice, and tumors were allowed to establish. Once the tumor size 272 reached approximately $100-120 \mathrm{~mm}^{3}$, mice were randomly segregated into the following 273 treatment groups: vehicle, AZD1208, docetaxel, and AZD1208 + docetaxel (Fig 6A).

274 Tumor volume was measured by caliper every $3^{\text {rd }}$ day until sacrifice. The effect on tumor 275 growth of either PIM inhibition or docetaxel alone was moderate, reducing overall tumor 276 growth by approximately $50 \%$ compared to control. Strikingly, combination therapy 277 produced a synergistic anti-tumor response that was significantly more effective than 278 either agent alone (Fig 6B and 6C). The effect of each treatment regimen on tumor cell 279 proliferation was assessed by immunohistochemical staining of Ki67. Although Ki67 280 staining was reduced in all treatment groups compared to vehicle, proliferation was 281 reduced to the greatest extent in animals treated with AZD1208 and docetaxel (Fig 6D 282 and 6E). Confirming our in vitro data that PIM inhibition induces apoptosis in docetaxel283 treated cells, cleaved caspase 3 (CC3) was increased by nearly 4-fold with combination 284 treatment compared to control or treatment with either agent alone (Fig 6D and 6E). To 285 confirm that the signaling axis between PIM and Drp1 is intact and effectively altering the 
286 mitochondrial phenotype in vivo, we first assessed Drp1 levels in each of our treatment 287 groups by immunohistochemistry. Treatment with docetaxel alone reduced Drp1 levels 288 compared to vehicle, whereas AZD1208 significantly increased Drp1 protein levels by over 289 3-fold (Fig 6E). Importantly, combination therapy significantly increased Drp1 levels 290 compared to AZD1208 alone. Next, tumor tissues ( $\mathrm{n}=3$ tumors/group) were lysed, and 291 immunoblotting was used to assess changes in Drp1 levels and phosphorylation status.

292 PIM inhibition alone or in combination with docetaxel, increased Drp1 phosphorylation at 293 S616 and decreased Drp1 phosphorylation at S637 (Fig 6F). Moreover, total levels of 294 Drp1 and CC3 were significantly increased in combination-treated tumors. To confirm that 295 PIM inhibition caused mitochondrial fragmentation in vivo, tumor tissues from control or 296 AZD1208-treated mice were processed and imaged by TEM. Significantly more 297 fragmented mitochondria were present in the AZD1208-treated tumors than the control 298 tumors (Fig 6G). Quantification revealed that the average mitochondrial length in tumors 299 was reduced from $1.1 \mu \mathrm{m}$ to $0.6 \mu \mathrm{m}$ with PIM inhibition (Fig $6 \mathrm{H}$ ). These findings indicate 300 that, PIM inhibition sensitizes NSCLC tumors to chemotherapy via the induction of 301 mitochondrial fragmentation, free radical generation, and apoptosis (Fig 6I).

303 Discussion: Due to the limited efficacy of chemotherapy in NSCLC patients, the 304 identification of new molecular targets to overcome drug resistance is central to improving 305 patient prognosis. Emerging clinical and preclinical evidence demonstrates that the 306 physical state of mitochondria significantly impacts the sensitivity of cancer cells to 307 chemotherapy. Therefore, identifying anti-cancer agents that alter mitochondrial dynamics 308 is a promising approach to overcome therapeutic resistance. Previous studies have shown 309 that one mechanism by which cisplatin and docetaxel induce cell death by is by enhancing 310 mitochondrial fragmentation and subsequent apoptosis $(27,28)$. In the past, activation of 311 PIM kinases has been implicated in chemoresistance via the phosphorylation of anti- 
312 apoptotic substrates, such as Bad and Bcl-2 (29,30). Here, we identify a new mechanism

313 by which PIM1 contributes to chemoresistance in NSCLC: by promoting mitochondrial 314 fusion.

315 The balance of mitochondrial fusion and fission affect mitochondrial function, which

316 can impact many cellular processes involved in tumorigenesis, including changes in

317 metabolism, proliferation, and apoptosis (31). However, the physiological consequences

318 of shifting mitochondrial dynamics depend on the extent of fusion/fragmentation as well

319 as environmental stress and genetic factors. For example, in RAS-driven pancreatic

320 cancers, mitochondrial fission is required for proliferation, and knockdown of Drp1 is

321 sufficient to block tumor growth, indicating a pro-tumorigenic role for fission $(9,32)$.

322 Alternatively, excessive mitochondrial fission in the same cell lines has been described to

323 induce oxidative injury, deplete mitochondrial ATP, and induce apoptosis (33). Moreover,

324 a fused mitochondrial network is correlated with cell survival and resistance to therapy in

325 many types of cancer, including $\operatorname{NSCLC}(7,34)$. Because Drp1 is required for mitochondrial

326 fission, understanding the cellular inputs that control its activation in cancer is critical for

327 predicting its impact on mitochondrial phenotype and the resulting changes in oxidative

328 stress and apoptosis. Drp1 activity is regulated by post-translational modification and

329 cellular localization. The ratio of phosphorylation at two critical regulatory sites on Drp1

330 (S616: activating and S637: inhibitory) controls its levels and localization to mitochondria.

331 Phosphorylation of Drp1 at S616 (activating) is mediated by two canonical pathways

332 associated with proliferation: ERK-Drp1 and RalA-RalBP1-Cdk1-Drp1 $(9,35)$, whereas,

333 phosphorylation of Drp1 at $\mathrm{S} 637$ (inhibitory) is modulated by pathways involved in

334 sensing cellular energy stress: AMPK-AKAP-PKA $(36,37)$. Interestingly, while the overall

335 trend is consistently toward activation of Drp1, the relative impact of PIM inhibition on total

336 levels of Drp1 and regulation of its phosphorylation status appears to differ in cell lines

337 from different types of cancer. Inactivation of PIM significantly altered phosphorylation at 
338 both sites, increasing the activating phosphorylation at S616 and decreasing the inhibitory 339 phosphorylation at S637. PIM1 overexpression was previously shown to increase 340 phospho-Drp1 (S637) in cardiomyocytes isolated from transgenic mice (38), and our data 341 corroborated these results in cancer cells. Thus, Drp1 is likely a direct target of PIM at the 342 inhibitory $\mathrm{S} 637$ site, which aligns with the protective role of PIM in cancer. We also 343 observed a marked increase in phospho-Drp1 (S616), which is typically associated with

344 ERK/CDK1activation. Indeed, Inhibition of PIM significantly upregulated ERK 345 phosphorylation compared to controls in a panel of lung cancer cell lines, whereas 346 overexpression of PIM1 decreased ERK phosphorylation (Fig. S6). This finding is 347 interesting, as crosstalk between PIM and ERK has not been previously described and 348 warrants further investigation.

349 Our data suggest that PIM1 expression is particularly relevant to the dysregulation

350 of apoptosis in NSCLC. PIM1 increases with disease progression in NSCLC and high 351 expression of PIM1 is significantly associated with worse overall survival. PIM kinases are 352 induced in response to cellular stress, including mechanical stress, nutrient deprivation, 353 and hypoxia $(20,39,40)$, so it is likely that changes in the tumor microenvironment account 354 for the observed increase in PIM1 in later stage NSCLC. Supporting prior studies, we 355 observe a significant increase in PIM1 levels following treatment with docetaxel in vitro 356 and in vivo, providing further evidence of its role as a key player in the cellular response 357 to stress (41). In vitro, overexpression of PIM1 decreased the sensitivity of NSCLC cell 358 lines to docetaxel and cisplatin. This finding is of particular significance in the context of 359 therapeutic resistance, as NSCLC patients with high PIM1 display a significantly worse 360 response to chemotherapy than patients with low PIM1 (Fig 1B). Here, we demonstrate 361 that PIM kinase inhibitors cause excessive mitochondrial fragmentation and triggered 362 superoxide overloading, leading to oxidative stress and reduction in the MMP. These 363 alterations were accompanied by an upregulation of proapoptotic proteins and a 
364 downregulation of antiapoptotic factors. Combined treatment with PIM inhibitors and 365 docetaxel or cisplatin produced a synergistic cytotoxic response in NSCLC in vitro and in 366 vivo. Moreover, PIM inhibitors sensitized NSCLC cell lines to chemotherapy in NSCLC 367 cell lines with WT Ras ( $\mathrm{NCl}-\mathrm{H} 2228)$ and mutant EGFR (NCl-H1975), indicating that this 368 therapeutic strategy irrespective of mutational status (Fig S7). Importantly, the dependent 369 on mitochondrial fragmentation, as stable knockdown of Drp1 in NSCLC cells blocked the 370 ability of PIM inhibitors to induce mitochondrial fragmentation and increase ROS. In these 371 cells, combining PIM inhibitors with chemotherapy was far less effective, demonstrating 372 that the impact of PIM on the mitochondrial phenotype is central to its utility as a 373 therapeutic target. It remains to be determined whether PIM also controls mitochondrial 374 dynamics in primary cells, and if so, whether this would limit the therapeutic window for 375 PIM inhibitors. Previous studies have described a role for PIM in regulating mitochondrial 376 dynamics in cardiomyocytes (38), so it is likely that PIM is a general mediator of 377 mitochondrial phenotype. However, PIM1 is upregulated in cancer and by many aspects 378 of the tumor microenvironment, such as hypoxia. Therefore, we predict that the effect of 379 PIM inhibitors on mitochondrial dynamics and ROS production would be more dramatic in 380 cancer cells compared to primary cells, providing some specificity toward tumor tissue.

381 Tumors are constantly exposed to environmental stress that result in ATP 382 depletion and increased oxidative stress. In order to adapt and survive, it is essential for 383 tumor cells to maintain redox homeostasis. Previous work from our lab and others 384 demonstrated that loss of PIM kinases reduces Nrf2 activity and lowers the antioxidant 385 capacity of cancer cells, leading to the accumulation of ROS $(42,43)$. Here, we describe a 386 new role for PIM kinases in controlling redox homeostasis in cancer by controlling 387 mitochondrial dynamics and increasing the production of ROS, independent of their ability 388 to alter Nrf2 and the anti-oxidant response. Based on these findings, we propose that PIM 389 serves a dual role in controlling oxidative stress. First, PIM maintains an elongated 
390 mitochondrial network, limiting acute ROS production at the mitochondria. At the same

391 time, increased PIM stabilizes Nrf2 to increase the antioxidant capacity of the cell. Thus,

392 inhibition of PIM both stimulates the production of ROS at the mitochondria by causing

393 excessive fission and also decreases the antioxidant response by reducing Nrf2 activation.

394 Together, these effects place cancer cells into a state of severe oxidative stress and

395 render them hypersensitive to anti-cancer therapy.

396

397 
Materials and Methods:

399 Plasmids: pCIP and hPIM1 constructs were created by subcloning into the expression

400 vector pCIG3 (pCMV-IRES-GFP, a gift from Dr. Felicia Goodrum, Addgene, plasmid

401 \#78264), modified to replace the GFP cassette with puromycin resistance gene. pSuper-

402 Retro-puro-human shDrp1 was previously described (9).

403

404 Reagents and antibodies: AZD1208 was acquired from AdooQ Biosciences. Docetaxel

405 and Mdivi-1 were obtained from Selleck Chemicals. JC-1, mitoSOX-red and $\mathrm{H}_{2}$-DCFDA

406 were purchased from Invitrogen. Primary antibodies for immunoblot and

407 immunofluorescence analyses were purchased from BD Biosciences [Actin, 612656],

408 Santa Cruz [Drp1, sc-271583, PIM1, sc-13513 and Bcl-2, sc-7382], and Cell Signaling

409 Technology [TOM20, 4240S; pIRS (S1101), 2385S; PIM1, 3247S; pDRP1 (S616), 3455S;

410 pDRP1 (S637), 4867S; and cleaved caspase-3, 9661S. All other materials and chemicals

411 were of reagent grade.

412

413 Cell transfection and immunoblotting: Wild type (WT) mouse embryonic fibroblasts

414 (MEFs), triple-knockout (TKO; Pim1 ${ }^{-/-}, \mathrm{Pim}^{-/-}$, and $\mathrm{Pim}^{-/-}$) MEFs (21), TKO MEFs stably

415 expressing PIM1 (TKO-PIM1) (22), H1299, H1299Keap1 ${ }^{-/-}$, A549, and H460 cells were

416 maintained in DMEM containing 10\% FBS and 1\% penicillin/streptomycin. All cell lines

417 were maintained at $37^{\circ} \mathrm{C}$ in $5 \% \mathrm{CO}_{2}$ and were authenticated by short tandem repeat DNA

418 profiling performed by the University of Arizona Genetics Core Facility. The cell lines were

419 used for fewer than 50 passages and routinely tested for mycoplasma contamination.

420 Stable retroviral transfections were carried out to generate PIM1 overexpressing (hPIM1)

421 and Drp1 knockout (shDrp1) cells. Immunoblotting was performed as described previously

422 (23).

423 
424 Mitochondrial superoxide assay: MitoSOX red staining was performed to measure

425 mitochondrial superoxide production. Cells were plated in 6-well plates containing

426 microscope coverslips and treated as indicated. Post-treatment, cells were incubated with

$4275 \mu \mathrm{M}$ MitoSOX red dissolved in Hank's Balanced Salt Solution (HBSS) for 30 minutes.

428 Then, cells were washed with 1X DPBS and associated staining was determined either

429 on mounted slides or in live cells using fluorescent microscopy.

430

431 ROS assay: Electron paramagnetic resonance (EPR) was used to measure ROS in $432 \mathrm{H} 1299, \mathrm{~A} 549$, and H460 cells treated with PIM447 for 48 h. Post-treatment, cells were 433 incubated in Krebs-HEPES buffer, pH 7.4 containing $200 \mu \mathrm{M} \mathrm{CMH}$ (1-hydroxy-3434 methoxycarbonyl-2,2,5,5-tetramethylpyrrolidine) probe for 30 minutes at $37^{\circ} \mathrm{C}$. Cells 435 treated with $500 \mu \mathrm{M}$ hydrogen peroxide for the same incubation period served as a positive 436 control. Fifty microliters of the Incubation solution were added to a glass EPR capillary 437 tube (Noxygen Science Transfer \& Diagnostics, Elzach, Germany) that was placed inside 438 the cavity of the E-scan spectrometer for data acquisition. The parameter settings for 439 acquisition were as follows: center field $1.99 \mathrm{~g}$, microwave power $1 \mathrm{~mW}$, modulation 440 amplitude 9 G, sweep time 10 seconds, number of scans 10, and field sweep $60 \mathrm{G}$. 441 Sample temperature was stabilized and kept at $37^{\circ} \mathrm{C}$ by the Temperature \& Gas Controller

442 "Bio III" unit, interfaced to the spectrometer. Spectra were recorded and analyzed by using 443 Win EPR software (2.11 version). ROS was also measured by DCF staining, as described 444 previously (20).

446 Immunofluorescence: Cells were plated in 6-well plates containing microscope 447 coverslips and treated as indicated. Post-treatment, cells were fixed with $10 \%$ buffered 448 formalin for $20 \mathrm{~min}$ and kept in blocking solution (5\% NGS and 0.3\% TritonX-100 in PBS) 449 for $60 \mathrm{~min}$. Then, cells were incubated with anti-TOM20 (rabbit mAb, 1:500 dilution), or 
450 anti-PIM1 (mouse mAb, 1: 100 dilution) or anti-DRP1 (mouse mAb, 1:200 dilution)

451 antibodies for 60 min. Following primary antibody incubation, cells were washed with $1 \times$

452 PBS and incubated in secondary antibodies (Alexa Fluor 568 goat anti-mouse and Alexa

453 Fluor 488 goat anti-rabbit, 1:500 dilution) for $60 \mathrm{~min}$. Finally, cells were mounted on glass

454 slides with mounting media (Cell Signaling Technology 8961S, Prolong® Gold) containing

455 DAPI. Images were taken at 60× magnification using a fluorescent microscope.

457 Transmission electron microscopy: Cells or tumor tissue samples were fixed with $2.5 \%$

458 glutaraldehyde in $0.1 \mathrm{M} \mathrm{PIPES}$ buffer, $\mathrm{pH} 7.4$ overnight at $4^{\circ} \mathrm{C}$. The samples were then

459 washed with 0.1M PIPES, $\mathrm{pH} 7.4$ three times for 10 minutes each. The samples were then

460 post-fixed with $1 \%$ osmium tetroxide in PIPES, $\mathrm{pH} 7.4$ for $1 \mathrm{~h}$, washed with deionized water

461 two times for $10 \mathrm{~min}$, followed by $20 \mathrm{~min}$ in aqueous $2 \%$ uranyl acetate, and washed again

462 with deionized water for $10 \mathrm{~min}$. The samples were then dehydrated with a graded series

463 of increasing concentrations of ethanol $(50 \%, 70 \%, 90 \%$, and $100 \%)$ in Pelco Biowave

464 Pro microwave, set at $250 \mathrm{~W}, 20^{\circ} \mathrm{C}$, and vacuum for 40 seconds. The samples were then

465 infiltrated (microwave, 1:1 Spurr's resin ethanol, $250 \mathrm{~W}, 20^{\circ} \mathrm{C}$, vacuum 3 minutes and

466 Spurr's resin, $25 \mathrm{~W}, 20^{\circ} \mathrm{C}$ vacuum twice three minutes each) and embedded in Spurr's

467 resin overnight at $60^{\circ} \mathrm{C}$. Ultrathin $(60 \mathrm{~nm})$ sections were cut onto uncoated copper mesh

468 grids and stained with $2 \%$ lead acetate for 2 min. The samples were examined using FEl

$469 \mathrm{CM} 12$ transmission electron microscope operated at 80kV. Digital images were obtained

470 in 8-bit TIFF format using a $4 \times 4$ digital camera.

472 Cell viability assay and drug interaction assessment: The cell viability was measured

473 by crystal violet staining. Briefly, cells were plated in 96 -well plates, treated with

474 inhibitor/drugs for $72 \mathrm{~h}$, fixed in $4 \%$ formaldehyde, and stained with $0.1 \%$ crystal violet.

475 The cells were lysed in a $1 \%$ sodium dodecyl sulfate solution, and absorbance was 
476 measured using microplate reader at a wavelength of $595 \mathrm{~nm}$. Synergy between PIM

477 inhibitor and chemotherapies was assessed by calculating the combination index $(\mathrm{Cl})$

478 value using CompuSyn software. $\mathrm{Cl}<1$ indicated synergy (the smaller the value, the

479 greater the degree of synergy), $\mathrm{Cl}=1$ indicated an additive effect, and $\mathrm{Cl}>1$ indicated 480 antagonism.

481

482 JC-1 assay: Mitochondrial membrane potential (MMP) was determined by JC-1 assay.

483 A549 and H460 cells were plated in 96-well plates and allowed to adhere overnight. Then,

484 the cells were washed once with $1 \times$ DPBS, stained, and treated as indicated. At the end

485 of the treatment period, absorbance was recorded using a fluorescence microplate reader

486 with an excitation of $485 \mathrm{~nm}$ and emissions of $540 \mathrm{~nm}$ and $590 \mathrm{~nm}$. Hydrogen peroxide

487 was used as a positive control. MMP was determined using the ratio of the fluorescence

488 of J-aggregates $(590 \mathrm{~nm})$ to monomers $(540 \mathrm{~nm})$ and normalized to the respective DMSO

489 control. For JC-1 imaging, cells were seeded on 6-well plate, treated as indicated and

490 processed as described above. Finally, images were taken for green and red channels at

$49120 \times$ magnification using a fluorescent microscope.

493 In vivo studies: The sample size justification of 4 mice (8 tumors) per group is based on 494 comparing the combination to each individual agent alone. We will have $80 \%$ statistical 495 power to detect a standardized decrease of 1.325 between groups (difference divided by 496 standard deviation) assuming a two-sided alpha of 0.05. Five million A549 cells in PBS 497 were injected subcutaneously into each flank of SCID mice in PBS. Once average tumor 498 size reached approximately $100 \mathrm{~mm}^{3}$, mice were randomized for treatment with vehicle 499 (Cremophore EL/Ethanol/PBS-24/6/70 ratio, p.o. daily; 5\% DMSO + 30\% PEG + 5\% 500 Tween80 $+\mathrm{ddH}_{2} \mathrm{O}$, i.p. every $3^{\text {rd }}$ day), AZD1208 (30mg/kg by p.o. daily), docetaxel ( 5 $501 \mathrm{mg} / \mathrm{kg}$ by i.p. every $3^{\text {rd }}$ day), or AZD1208 + docetaxel. Tumor volume was monitored by 
502 caliper measurements. Thirty-six days after injection, animals were sacrificed, and tumors

503 were harvested. Tumors were fixed, embedded in paraffin, and sectioned for staining with

504 hematoxylin and eosin (H\&E) or antibodies specific for Ki67, Cleaved caspase-3 (CC3),

505 PIM1, and DRP1. Percent positive staining for the above mentioned proteins was

506 calculated using ImageJ analysis software. Investigators were blinded to the sample

507 information prior to software-based analysis. For Transmission electron microscopy (TEM)

508 analysis, tumors from vehicle and AZD1208-treated mice were fixed in $2.5 \%$

509 glutaraldehyde. All animal studies were approved by the Institutional Animal Care and Use

510 Committee at the University of Arizona.

511

512 Statistical analysis: Tumor growth was analyzed by fitting a mixed linear model of tumor

513 volume vs. time for each mouse. The resulting slopes (growth rate) was compared using

514 a factorial model with vehicle, AZD alone, Docetaxel alone, and the AZD + Docetaxel dual

515 treatment. Differences in proliferation and apoptosis among treatment groups was

516 analyzed using linear mixed models adjusted for the correlation among measurements

517 within the same mouse. All immunofluorescence staining and western blots are

518 representative of at least three independent experiments. Differences across groups were

519 determined by unpaired 2-tailed Student's t-test. One-way analysis of variance (ANOVA)

520 was used to analyze differences between more than two groups across one timepoint. P

521 values were adjusted using Bonferroni's multiple comparison test. The data is presented

522 as the mean SD or mean SEM as indicated, and a p-value $<0.05$ was considered

523 statistically significant.

524

525 
526 Acknowledgements: We would like to thank Dr. Donna Zhang (University of Arizona) for

527 providing the $\mathrm{H} 1299 \mathrm{Keap}^{-1^{--}}$cell line and assistance with the acquisition and analysis of

528 EPR results. We thank Adam R. Kohr for his assistance with graphic design. The research

529 was supported by American Cancer Society grant RSG-16-159-01-CDD, American Lung

530 Association grant LCD-504131, and Department of Defense PCRP Award (W81XWH-19-

531 1-0455) to NAW. Cancer Center Support Grant P30CA023074 also provided support for

532 this research.

533

534 Conflict of Interest: The authors disclose no conflicts of interest

535

536 Role in the study:

537 Study concept and design: NAW, SSC

538 Acquisition of data: SSC, RKT, CCJ, ALC, NAW

539 Analysis and presentation of data: NAW, SSC

540 Material support: DFA

541 Study supervision: NAW

542 Funding: NAW

543

544 
545

546

547

548

549

550

551

552

553

554

555

556

557

558

559

560

561

562

563

564

565

566

567

568

569

570

571

572

573

574

575

576

577

578

579

580

581

582

583

584

585

586

587

588

589

\section{References:}

1 Zappa C, Mousa SA. Non-small cell lung cancer: current treatment and future advances. Transl Lung Cancer Res 2016; 5: 288-300.

2 Lin JJ, Shaw AT. Resisting Resistance: Targeted Therapies in Lung Cancer. Trends Cancer 2016; 2: 350-364.

3 Joseph B, Ekedahl J, Sirzen F, Lewensohn R, Zhivotovsky B. Differences in expression of pro-caspases in small cell and non-small cell lung carcinoma. Biochem Biophys Res Commun 1999; 262: 381-387.

4 Li X, You M, Liu YJ, Ma L, Jin PP, Zhou R et al. Reversal of the Apoptotic Resistance of Non-Small-Cell Lung Carcinoma towards TRAIL by Natural Product Toosendanin. Sci Rep 2017; 7: 42748.

5 Zhao J, Zhang J, Yu M, Xie Y, Huang Y, Wolff DW et al. Mitochondrial dynamics regulates migration and invasion of breast cancer cells. Oncogene 2013; 32: 4814-4824.

6 Xie LL, Shi F, Tan Z, Li Y, Bode AM, Cao Y. Mitochondrial network structure homeostasis and cell death. Cancer Sci 2018; 109: 3686-3694.

7 Kong B, Wang Q, Fung E, Xue K, Tsang BK. p53 is required for cisplatin-induced processing of the mitochondrial fusion protein L-Opa1 that is mediated by the mitochondrial metallopeptidase Oma1 in gynecologic cancers. J Biol Chem 2014; 289: 27134-27145.

8 Trotta AP, Chipuk JE. Mitochondrial dynamics as regulators of cancer biology. Cell Mol Life Sci 2017; 74: 1999-2017.

9 Kashatus JA, Nascimento A, Myers LJ, Sher A, Byrne FL, Hoehn KL et al. Erk2 phosphorylation of Drp1 promotes mitochondrial fission and MAPK-driven tumor growth. Mol Cell 2015; 57: 537-551.

10 Jezek J, Cooper KF, Strich R. Reactive Oxygen Species and Mitochondrial Dynamics: The Yin and Yang of Mitochondrial Dysfunction and Cancer Progression. Antioxidants (Basel) 2018; 7.

11 von Eyss B, Jaenicke LA, Kortlever RM, Royla N, Wiese KE, Letschert S et al. A MYC-Driven Change in Mitochondrial Dynamics Limits YAP/TAZ Function in Mammary Epithelial Cells and Breast Cancer. Cancer Cell 2015; 28: 743-757.

12 Qian W, Wang J, Roginskaya V, McDermott LA, Edwards RP, Stolz DB et al. Novel combination of mitochondrial division inhibitor 1 (mdivi-1) and 
590

591

592

593

594

595

596

597

598

599

600

601

602

603

604

605

606

607

608

609

610

611

612

613

614

615

616

617

618

619

620

621

622

623

624

625

626

627

628

629

630

631

632

633

634

635 platinum agents produces synergistic pro-apoptotic effect in drug resistant tumor cells. Oncotarget 2014; 5: 4180-4194.

13 Breckenridge DG, Stojanovic M, Marcellus RC, Shore GC. Caspase cleavage product of BAP31 induces mitochondrial fission through endoplasmic reticulum calcium signals, enhancing cytochrome c release to the cytosol. J Cell Biol 2003; 160: 1115-1127.

14 Estaquier J, Arnoult D. Inhibiting Drp1-mediated mitochondrial fission selectively prevents the release of cytochrome c during apoptosis. Cell Death Differ 2007; 14: 1086-1094.

15 Frank S, Gaume B, Bergmann-Leitner ES, Leitner WW, Robert EG, Catez F et al. The role of dynamin-related protein 1 , a mediator of mitochondrial fission, in apoptosis. Dev Cell 2001; 1: 515-525.

16 Lee YJ, Jeong SY, Karbowski M, Smith CL, Youle RJ. Roles of the mammalian mitochondrial fission and fusion mediators Fis1, Drp1, and Opa1 in apoptosis. Mol Biol Cell 2004; 15: 5001-5011.

17 Thomas KJ, Jacobson MR. Defects in mitochondrial fission protein dynaminrelated protein 1 are linked to apoptotic resistance and autophagy in a lung cancer model. PLoS One 2012; 7: e45319.

18 Roberts ER, Thomas KJ. The role of mitochondria in the development and progression of lung cancer. Comput Struct Biotechnol J 2013; 6: e201303019.

19 Cao L, Wang F, Li S, Wang X, Huang D, Jiang R. PIM1 kinase promotes cell proliferation, metastasis and tumor growth of lung adenocarcinoma by potentiating the c-MET signaling pathway. Cancer Lett 2019; 444: 116-126.

20 Warfel NA, Sainz AG, Song JH, Kraft AS. PIM Kinase Inhibitors Kill Hypoxic Tumor Cells by Reducing Nrf2 Signaling and Increasing Reactive Oxygen Species. Mol Cancer Ther 2016; 15: 1637-1647.

21 Mikkers H, Nawijn M, Allen J, Brouwers C, Verhoeven E, Jonkers J et al. Mice deficient for all PIM kinases display reduced body size and impaired responses to hematopoietic growth factors. Mol Cell Biol (Research Support, Non-U.S. Gov't) 2004; 24: 6104-6115.

22 Song JH, An N, Chatterjee S, Kistner-Griffin E, Mahajan S, Mehrotra S et al. Deletion of Pim kinases elevates the cellular levels of reactive oxygen species and sensitizes to K-Ras-induced cell killing. Oncogene 2014.

23 Warfel NA, Niederst M, Stevens MW, Brennan PM, Frame MC, Newton AC. Mislocalization of the E3 ligase, beta-transducin repeat-containing protein 1 
636

637

638

639

640

641

642

643

644

645

646

647

648

649

650

651

652

653

654

655

656

657

658

659

660

661

662

663

664

665

666

667

668

669

670

671

672

673

674

675

676

677

678

679

680

681
(beta-TrCP1), in glioblastoma uncouples negative feedback between the pleckstrin homology domain leucine-rich repeat protein phosphatase 1 (PHLPP1) and Akt.J Biol Chem (Research Support, N.I.H., Extramural Research Support, U.S. Gov't, Non-P.H.S.) 2011; 286: 19777-19788.

24 Song JH, Padi SK, Luevano LA, Minden MD, DeAngelo DJ, Hardiman G et al. Insulin receptor substrate 1 is a substrate of the Pim protein kinases. Oncotarget 2016.

25 Rambold AS, Kostelecky B, Elia N, Lippincott-Schwartz J. Tubular network formation protects mitochondria from autophagosomal degradation during nutrient starvation. Proc Natl Acad Sci U S A 2011; 108: 10190-10195.

26 Bordt EA, Clerc P, Roelofs BA, Saladino AJ, Tretter L, Adam-Vizi V et al. The Putative Drp1 Inhibitor mdivi-1 Is a Reversible Mitochondrial Complex I Inhibitor that Modulates Reactive Oxygen Species. Dev Cell 2017; 40: 583-594 e586.

27 Choi YM, Kim HK, Shim W, Anwar MA, Kwon JW, Kwon HK et al. Mechanism of Cisplatin-Induced Cytotoxicity Is Correlated to Impaired Metabolism Due to Mitochondrial ROS Generation. PLoS One 2015; 10: e0135083.

28 Cristofani R, Montagnani Marelli M, Cicardi ME, Fontana F, Marzagalli M, Limonta $\mathrm{P}$ et al. Dual role of autophagy on docetaxel-sensitivity in prostate cancer cells. Cell Death Dis 2018; 9: 889.

29 Lilly M, Sandholm J, Cooper JJ, Koskinen PJ, Kraft A. The PIM-1 serine kinase prolongs survival and inhibits apoptosis-related mitochondrial dysfunction in part through a bcl-2-dependent pathway. Oncogene 1999; 18: 4022-4031.

30 Macdonald A, Campbell DG, Toth R, McLauchlan H, Hastie CJ, Arthur JS. Pim kinases phosphorylate multiple sites on Bad and promote 14-3-3 binding and dissociation from Bcl-XL. BMC Cell Biol 2006; 7: 1.

31 Westermann B. Mitochondrial fusion and fission in cell life and death. Nat Rev Mol Cell Biol 2010; 11: 872-884.

32 Nagdas S, Kashatus JA, Nascimento A, Hussain SS, Trainor RE, Pollock SR et al. Drp1 Promotes KRas-Driven Metabolic Changes to Drive Pancreatic Tumor Growth. Cell Rep 2019; 28: 1845-1859 e1845.

33 Wan J, Cui J, Wang L, Wu K, Hong X, Zou Y et al. Excessive mitochondrial fragmentation triggered by erlotinib promotes pancreatic cancer PANC-1 cell apoptosis via activating the mROS-HtrA2/Omi pathways. Cancer Cell Int 2018; 18: 165. 
68234 Senft D, Ronai ZA. Regulators of mitochondrial dynamics in cancer. Curr Opin

$683 \quad$ Cell Biol 2016; 39: 43-52.

684

68535 Kashatus DF, Lim KH, Brady DC, Pershing NL, Cox AD, Counter CM. RALA and

686

687 RALBP1 regulate mitochondrial fission at mitosis. Nat Cell Biol 2011; 13: 1108-

688

689 1115.

690

36 Chang CR, Blackstone C. Cyclic AMP-dependent protein kinase

691

692

693

37 Cribbs JT, Strack S. Reversible phosphorylation of Drp1 by cyclic AMP-

694

695 dependent protein kinase and calcineurin regulates mitochondrial fission and

696

697

38 Din S, Mason M, Volkers M, Johnson B, Cottage CT, Wang Z et al. Pim-1

698 phosphorylation of Drp1 regulates its GTPase activity and mitochondrial morphology. J Biol Chem 2007; 282: 21583-21587.

699

700

701

39 Warfel NA, Kraft AS. PIM kinase (and Akt) biology and signaling in tumors. Pharmacol Ther 2015; 151: 41-49.

703

704

40 Tu ML, Wang HQ, Sun XD, Chen LJ, Peng XC, Yuan YH et al. Pim-1 is upregulated by shear stress and is involved in shear stress-induced proliferation

705

706

707

708

709

710

711

712

713

42 Song JH, Singh N, Luevano LA, Padi SKR, Okumura K, Olive V et al. Mechanisms

714 of rat mesenchymal stem cells. Life Sci 2011; 88: 233-238.

41 Zemskova M, Sahakian E, Bashkirova S, Lilly M. The PIM1 kinase is a critical component of a survival pathway activated by docetaxel and promotes survival of docetaxel-treated prostate cancer cells. J Biol Chem 2008; 283: 20635-20644.

715

716

717

718

719

720

721

722

Behind Resistance to PI3K Inhibitor Treatment Induced by the PIM Kinase. Mol Cancer Ther 2018; 17: 2710-2721. 
723 Figure 1. PIM1 is upregulated in advanced lung cancer and predicts poor survival

724 outcomes: (A) Representative immunohistochemical staining of PIM1 expression by 725 clinical stage in human lung cancer tissue array (main section, scale bars $100 \mu \mathrm{m}$; inset, 726 scale bars $20 \mu \mathrm{m}$ ). (B) Quantification of average PIM1 expression in stage II vs. stage III 727 of human lung cancer, mean \pm SEM, $n=37$. ${ }^{* * *}, p<0.001$. (C) Kaplan-Meier analysis of 728 overall survival in human lung cancer adenocarcinoma patients with high vs. low PIM1, 729 and (D) overall survival of patients treated with chemotherapy with high vs. low PIM1.

731 Figure 2. PIM inhibition increases mitochondrial superoxide production and total 732 cellular ROS: (A) Mitochondrial ROS was detected by MitoSOX staining in WT, TKO, and 733 TKO-PIM1 MEFs and (B) WT MEFs treated with PIM447 (3 $\mu \mathrm{M})$ for $24 \mathrm{~h}$. (C) The indicated 734 lung cancer cells were treated with AZD1208 $(5 \mu \mathrm{M})$ for $24 \mathrm{~h}$, and (D) MitoSOX staining 735 was quantified as the fold change in CTCF. Representative micrographs of live cell 736 imaging of MitoSOX (red) staining in lung cancer cells treated with AZD1208 (5 $\mu$ M) for 2

737 h. (E) Fold change in ROS levels as measured by EPR in lung cancer cells treated as 738 indicated; ROS levels, $\mathrm{nM} / \mathrm{min} / \mathrm{mg}$ protein; mean $\pm \mathrm{SD}$ of three independent experiments. 739 **, p<0.01 vs WT MEFs; ${ }^{*}, p<0.01$ vs TKO MEFs; ${ }^{*}, p<0.05,{ }^{* *}, p<0.01,{ }^{* * *}, p<0.001$ vs 740 DMSO. Scale bars, $20 \mu \mathrm{m}$.

742 Figure 3. Loss of PIM induces mitochondrial fragmentation: (A) Representative 743 western blots showing PIM levels and activity in MEFs. (B) Mitochondrial phenotype was 744 assessed by TOM20 immunofluorescence (green), (C) and fragmented mitochondria (<1 $745 \mu \mathrm{m}$ ) were quantified (red arrowheads and mitochondria within red dotted line indicate 746 fragmented mitochondria. (D-F) A549 cells were treated with PIM447 (3 $\mu \mathrm{M})$ for $24 \mathrm{~h}$ and 747 mitochondrial phenotype was assessed by TOM20 staining. (G) H1299 cells were treated 748 with AZD1208 $(5 \mu \mathrm{M})$ for $24 \mathrm{~h}$, and $(\mathrm{H})$ mitochondria were imaged by TEM. (I) Percentage 
749 of mitochondria with elongated (> $1.5 \mu \mathrm{m}$, yellow), intermediate $(1-1.5 \mu \mathrm{m}$, blue) and

750 fragmented $(<1 \mu \mathrm{m}$, red) mitochondria were quantified. Results are expressed as mean

$751 \pm$ SD of three independent experiments. *,$p<0.05$ vs WT MEFs; \#, $p<0.05$ vs TKO MEFs;

$752{ }^{*}, p<0.05,{ }^{* *}, p<0.01,{ }^{* *}, p<0.001$ vs DMSO. Scale bars, $20 \mu \mathrm{m}$.

753

754 Figure 4. PIM1 affects mitochondrial phenotype in a Drp1-dependent manner: (A)

755 Representative immunohistochemical staining and (B) quantification of PIM1 and Drp1 in

756 NSCLC TMA (main section, scale bars $100 \mu \mathrm{m}$; inset, scale bars $20 \mu \mathrm{m}$ ). (C) Drp1 levels

757 and phosphorylation were assessed by Immunoblotting in MEFs and lung cancer cell lines

758 treated with DMSO or PIM447 $(3 \mu \mathrm{M})$ for 24 h. (D) TKO MEFs were treated with a DRP1

759 inhibitor (Mdivi-1) and or HBSS and mitochondrial phenotype was assessed by TOM20

760 staining (green). (E) A549 cells were treated with the indicated drugs and mitochondrial

761 phenotype was assessed by TOM20 staining (scale bars, $20 \mu \mathrm{m}$; ${ }^{* * *}, \mathrm{p}<0.001$ vs DMSO;

762 and ${ }^{\# \#}, p<0.001$ vs PIM447). (F) Immunoblot and (G) Immunofluorescence analysis of

763 TOM20 in Drp1 knockdown lung cancer cells treated with PIM447 (3 $\mu \mathrm{M})$ for $24 \mathrm{~h}$;

764 Magnification 60X; scale bars, $20 \mu \mathrm{m} ; .{ }^{*}, \mathrm{p}<0.05 ;{ }^{* * *}, \mathrm{p}<0.001$ vs DMSO treated parental

765 line; \#\#, p<0.001 vs PIM447-treated parental line. (H) WT, TKO, and TKO-PIM1 MEFs

766 were stained for TOM20 (green) and Drp1 (red) and co-localization was quantified as the

767 fold change in co-localized pixels (yellow); ${ }^{* * *}, p<0.01$ vs WT MEFs; ${ }^{\# \#, ~} p<0.01$ vs TKO

768 MEFs). Values are mean \pm SD of 3 independent experiments. Scale bars, $20 \mu \mathrm{m}$.

769

770 Figure 5. PIM inhibition sensitizes lung cancer cells to chemotherapy by altering the

771 mitochondrial phenotype. Stable PIM1 overexpression in lung cancer cells confirmed

772 by (A) western blotting and (B) Immunofluorescence analyses. (C) Mitochondrial

773 phenotype assessed by TOM20 immunofluorescence (green) in A549 and H460 cells with 
774 PIM1 overexpression. Magnification 60X; scale bars, $20 \mu \mathrm{m} .\left(^{*}, p<0.05 ;{ }^{* *}, p<0.01\right.$ vs pCIP

775 transfected line). (D) Crystal violet cell viability assay and drug IC50 quantification in lung

776 cancer cells after $72 \mathrm{~h}$ docetaxel or cisplatin treatment. (E) Flow cytometric analysis of $\mathrm{H}_{2^{-}}$

777 DCFDA intensity representing ROS levels in A549 and H460 cells treated with PIM447,

778 docetaxel, or the combination. (F) Immunoblot analysis of A549 and H460 cells treated

779 with PIM447 (3 $\mu \mathrm{M})$, docetaxel (3 nM), or the combination. (G) Live cell micrographs of

780 JC-1 aggregates in A549 and H460 cells treated with PIM447, docetaxel, or the

781 combination (Scale bars, $40 \mu \mathrm{m}$ ), and $(\mathrm{H}) \mathrm{JC}-1$ staining was quantified using a

782 fluorescence plate reader. Values are mean \pm SD of 3 independent experiments. **, $783 \mathrm{p}<0.01,{ }^{* * *}, p<0.001$ vs DMSO; $, p<0.05,{ }^{\# \#}, p<0.01,{ }^{\# \#}, p<0.001$ vs PIM447; and ${ }^{\dagger+\dagger}$, $784 p<0.001$ vs Docetaxel.

785

786 Figure 6. Combined treatment with PIM1 inhibitor and docetaxel displays 787 synergistic anti-tumor effects in vivo. (A) Schematic of experimental design. (B)

788 Representative tumors isolated from each treatment group $(n=6)$. (C) Tumor volume $789\left(\mathrm{~mm}^{3}\right)$ was determined over time (values $\pm \mathrm{SD} ;{ }^{* * *}, p<0.001$ vs vehicle; ${ }^{\# \#,}, p<0.001$ vs 790 AZD1208; and ${ }^{\dagger+\dagger}, p<0.001$ vs docetaxel). (D) Representative images of tumor sections (4 $791 \mu \mathrm{m}$ ) examined by $\mathrm{H} \& \mathrm{E}$ and immunohistochemical analysis (scale bars, $20 \mu \mathrm{m}$ ). (E)

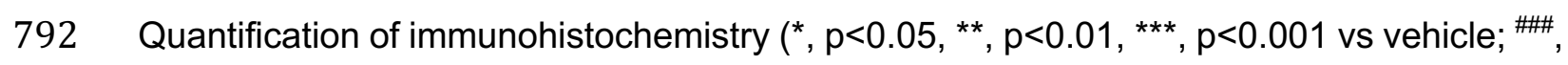
$793 p<0.001$ vs AZD1208; and ${ }^{\dagger \dagger}, p<0.01,{ }^{+\dagger}, p<0.001$ vs docetaxel). (F) Immunoblot analysis 794 of tumor samples isolated from each group $(n=3) .(G)$ Tumors isolated from control and 795 AZD1208-treated mice were imaged by TEM, and $(H)$ mitochondrial length was quantified 796 (scale bars, $500 \mathrm{~nm})\left(\mathrm{n}=49\right.$; values \pm SEM; ${ }^{* * *}, \mathrm{p}<0.001$ vs vehicle). (I) Model describing 797 the role of PIM in regulating the mitochondrial phenotype, ROS production and 798 survival/therapeutic resistance. 
A.

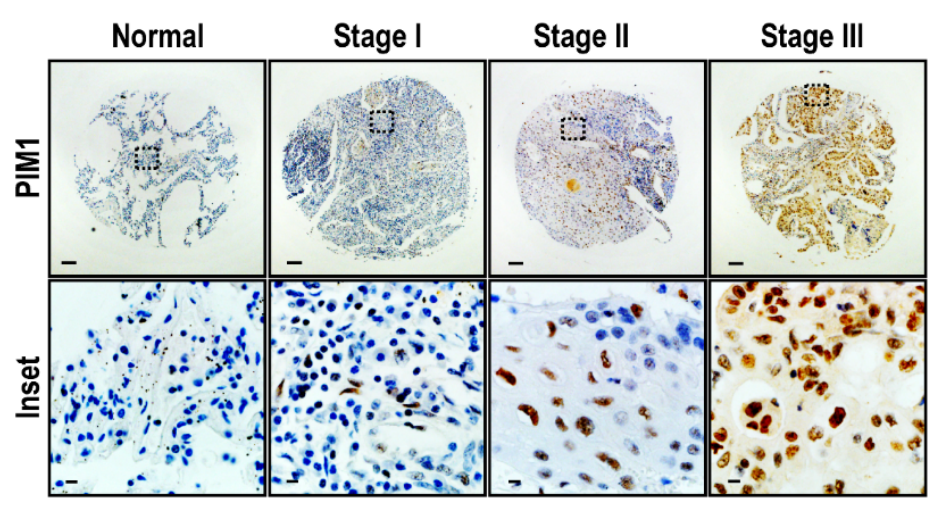

Fig 1

B.

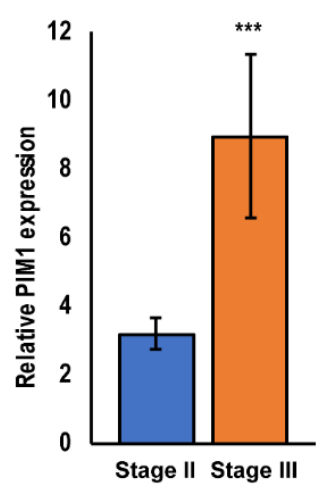

C.

PIM1 vs Lung cancer adenocarcinoma
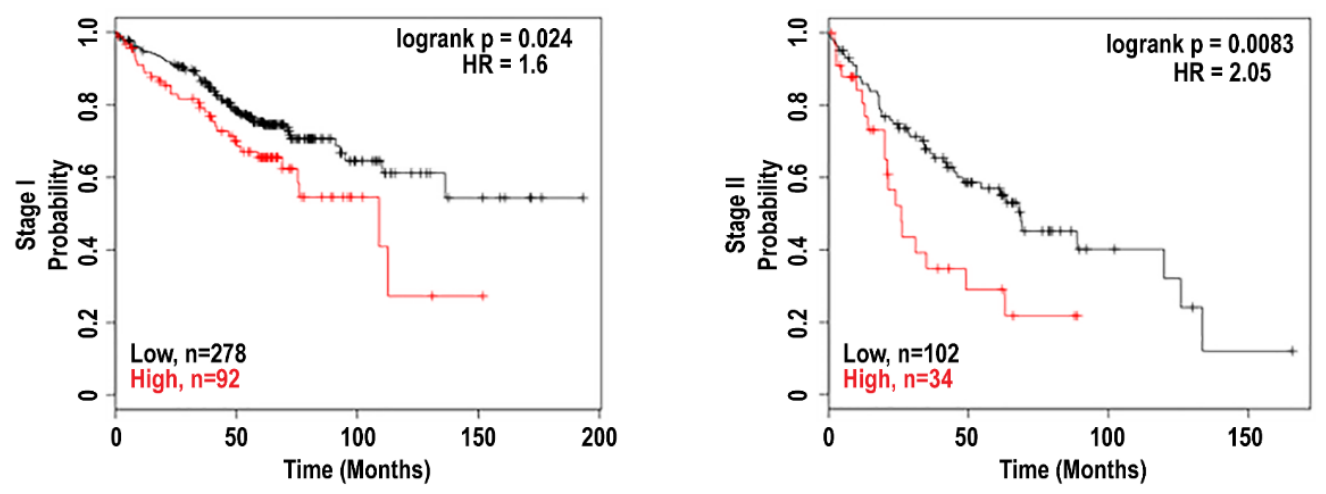

D.
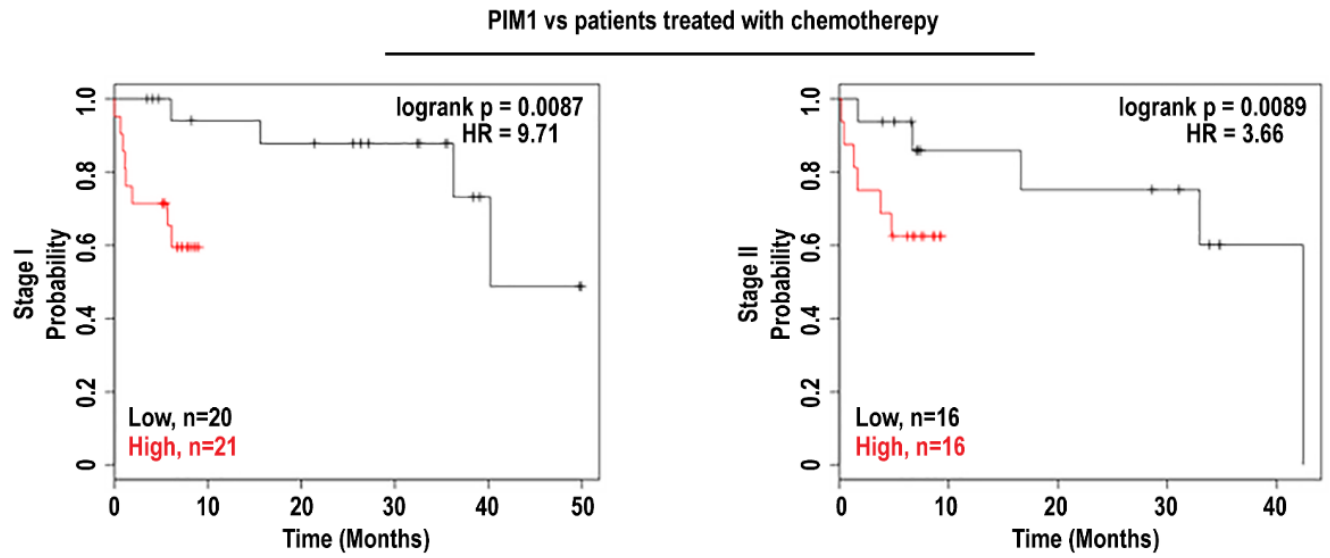
A.

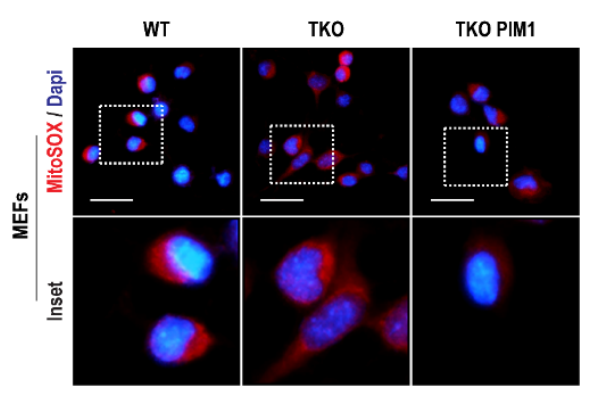

c.

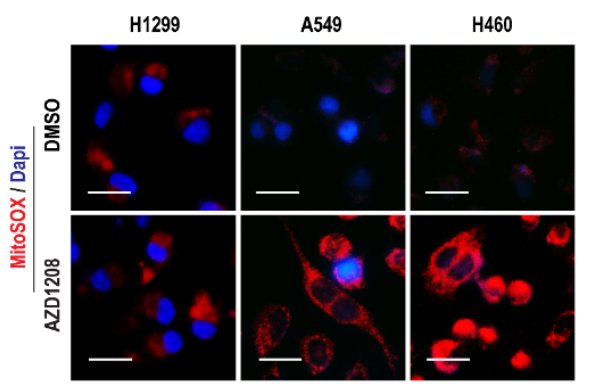

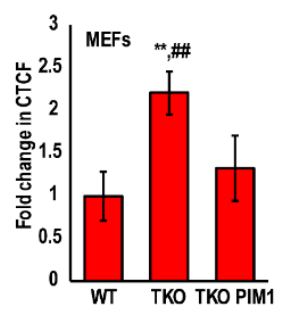

B.

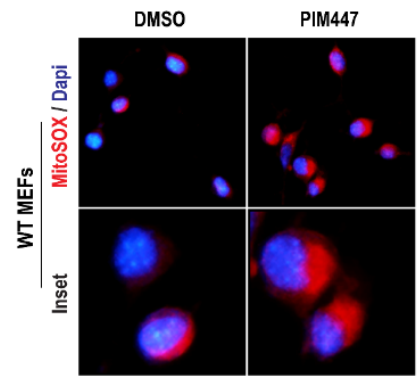

Fig 2

D.

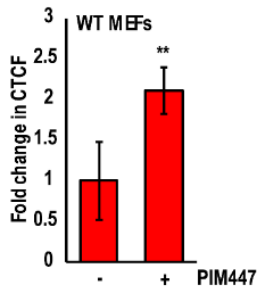

E.
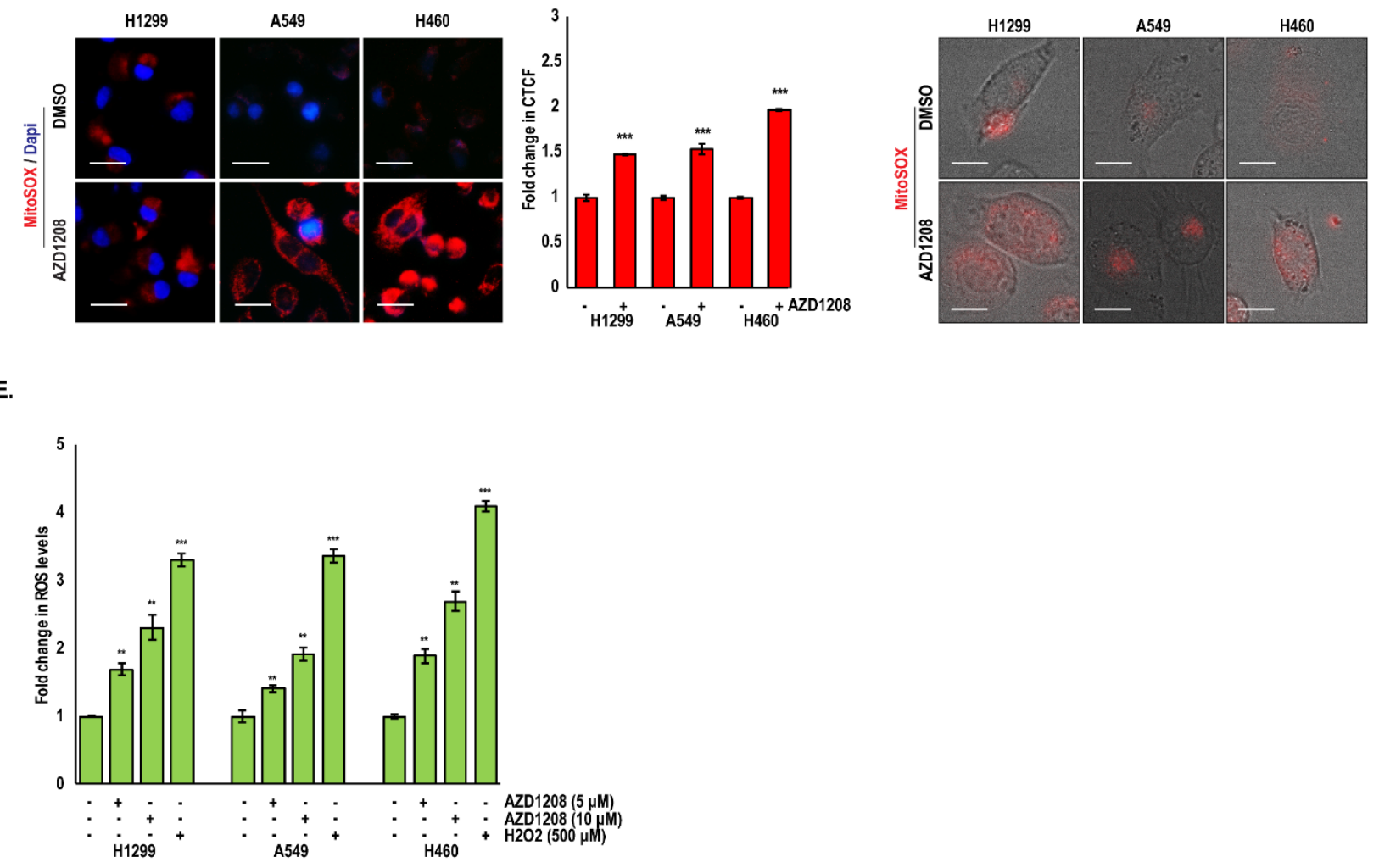
Fig 3

A.

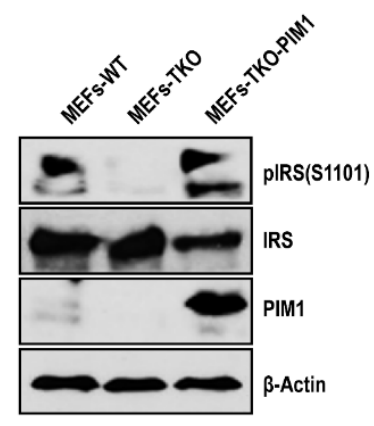

D.

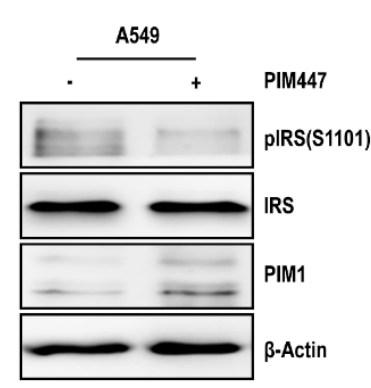

G.

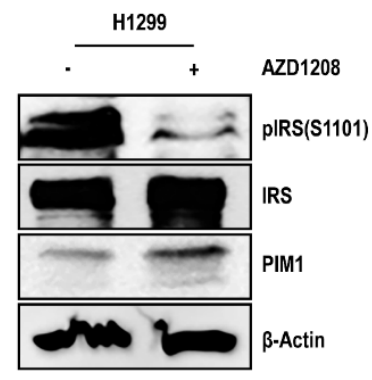

B.

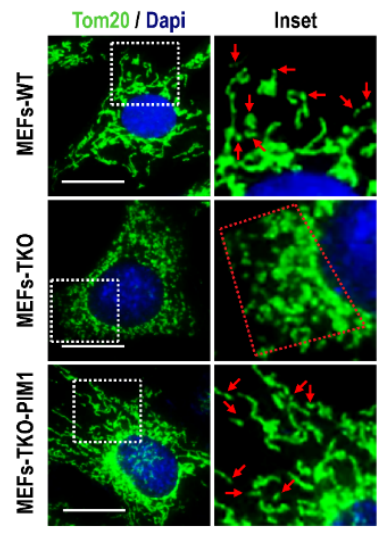

E.

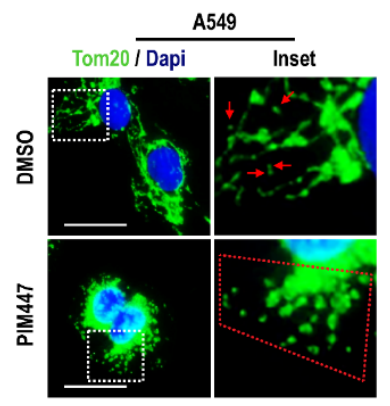

H.

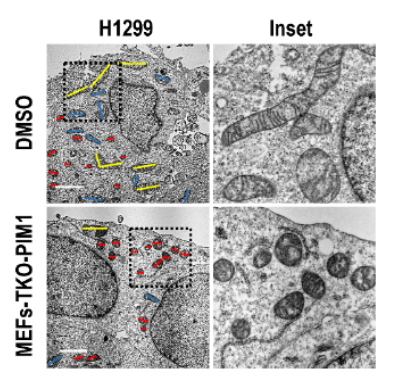

C.

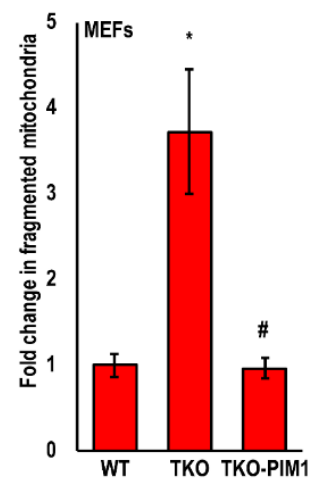

F.

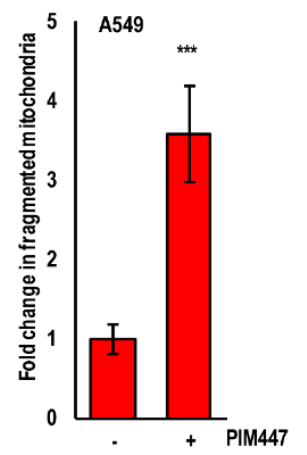

I.

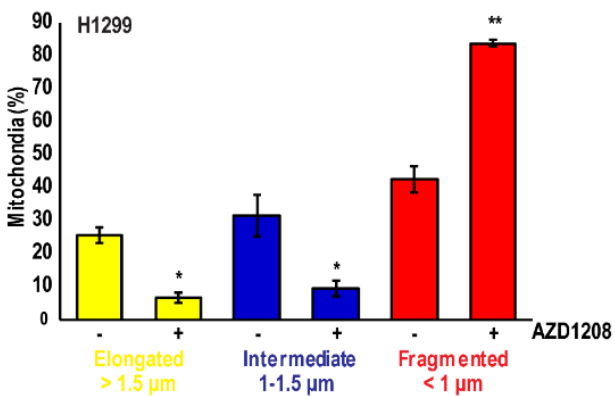


A.

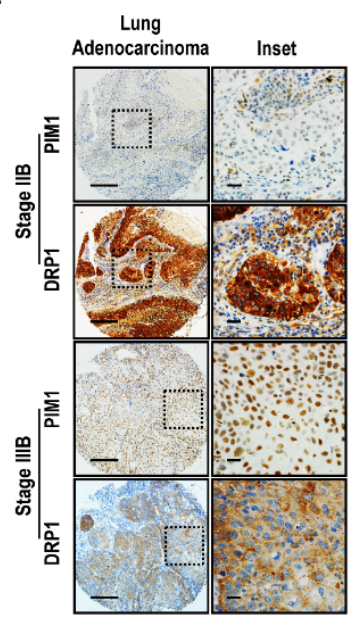

B.

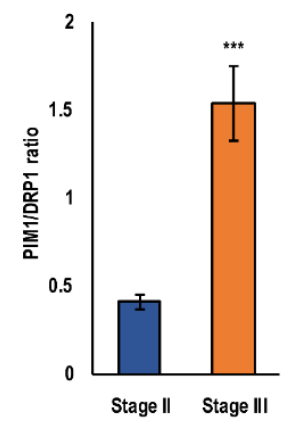

C.

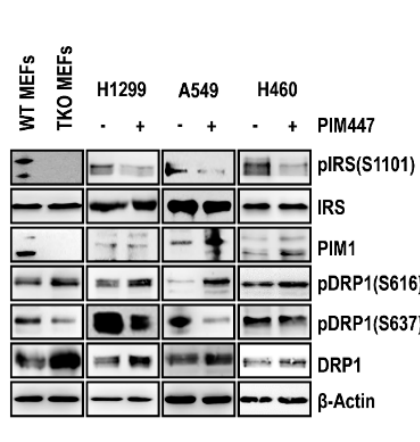

D.

E.

F.

H.
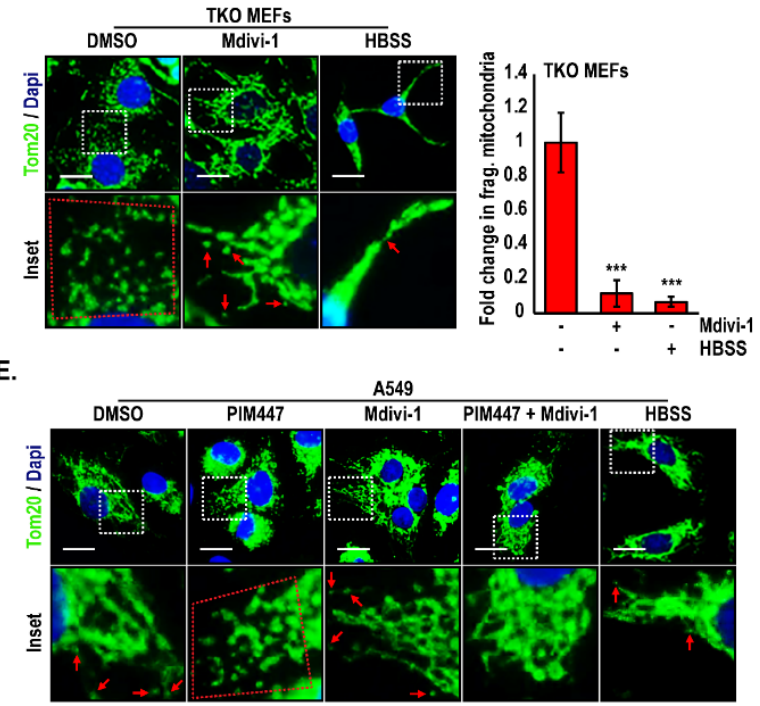

G.
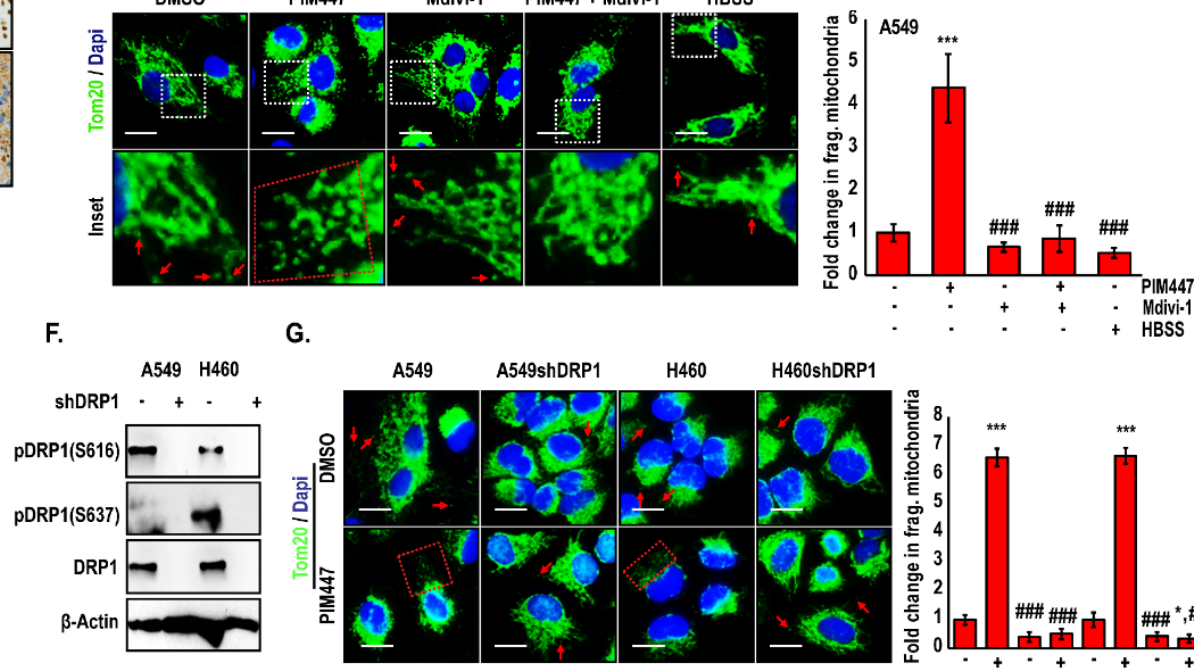

Fig 4
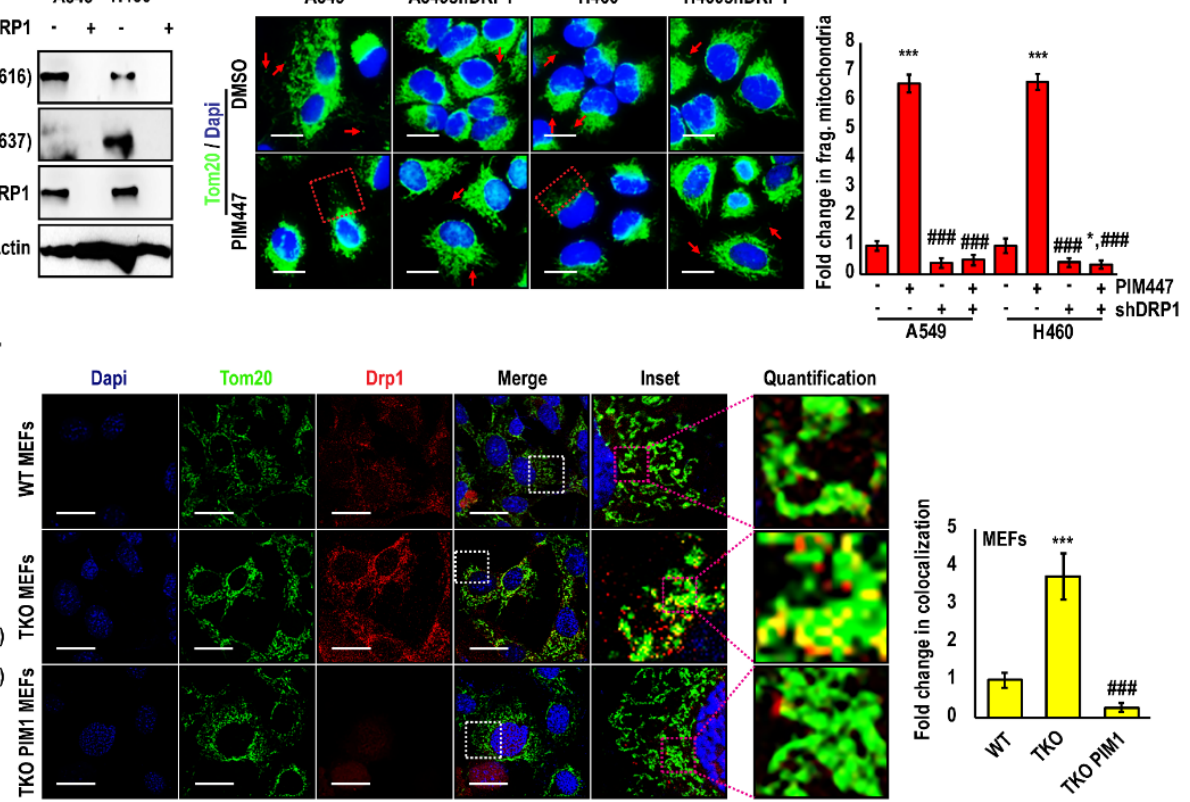
A.
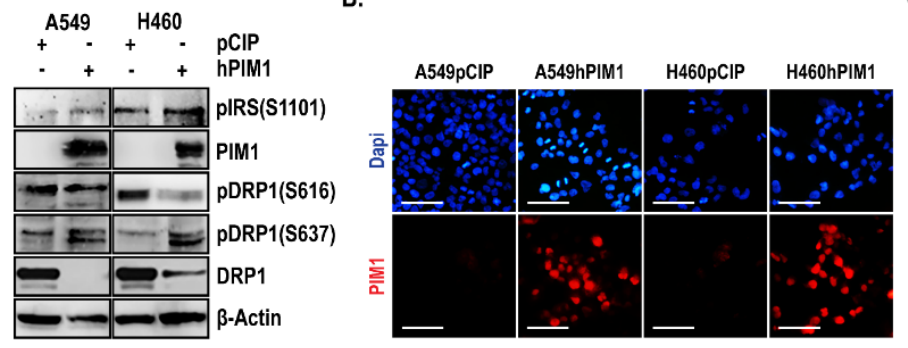

D.
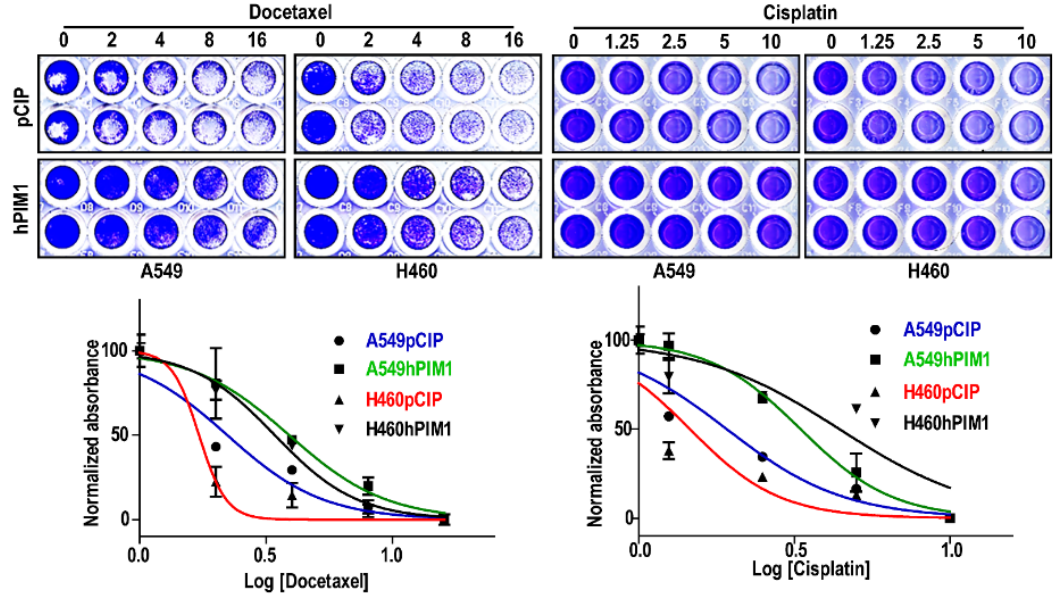

F.

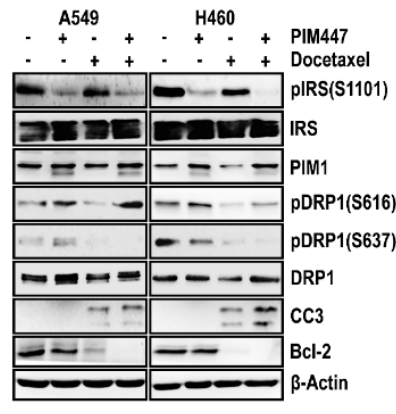

G.

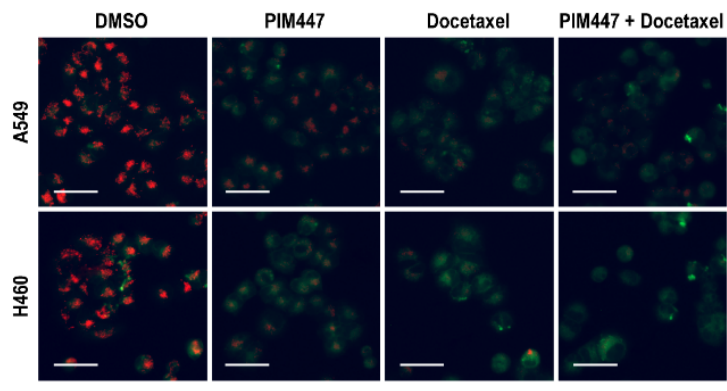

C.

Fig 5

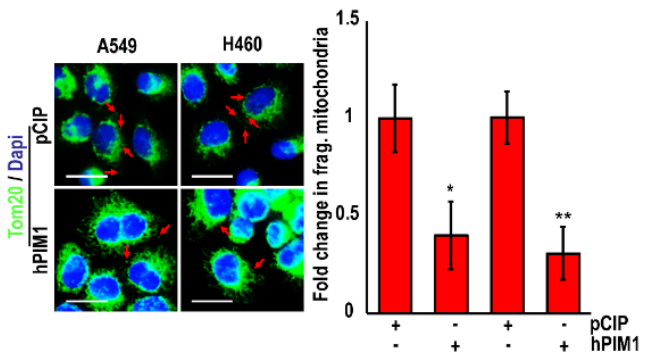

E.
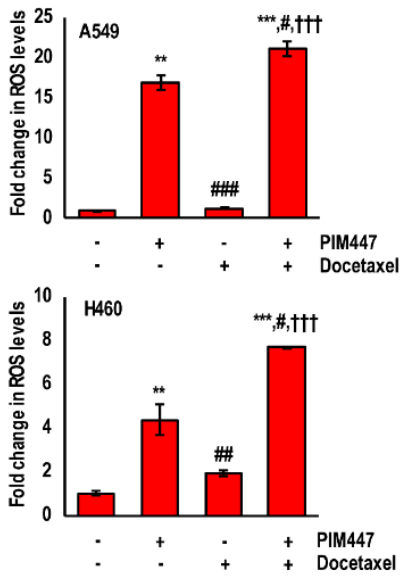

H.

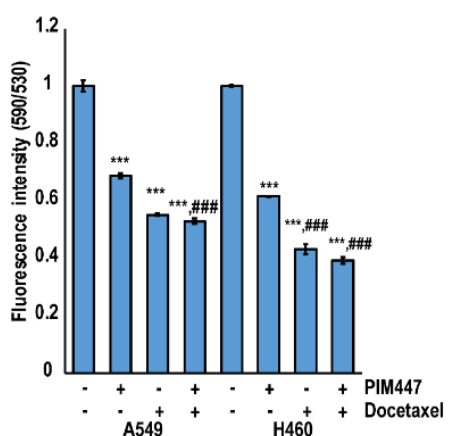


A.

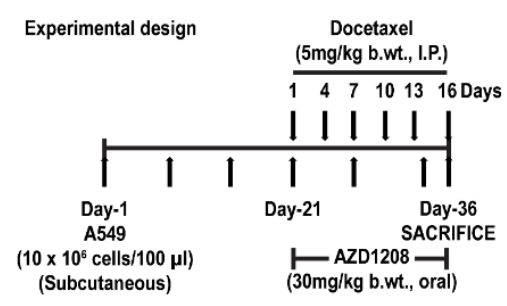

B.

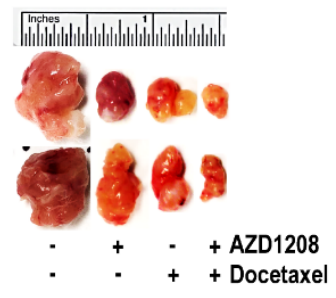

D.

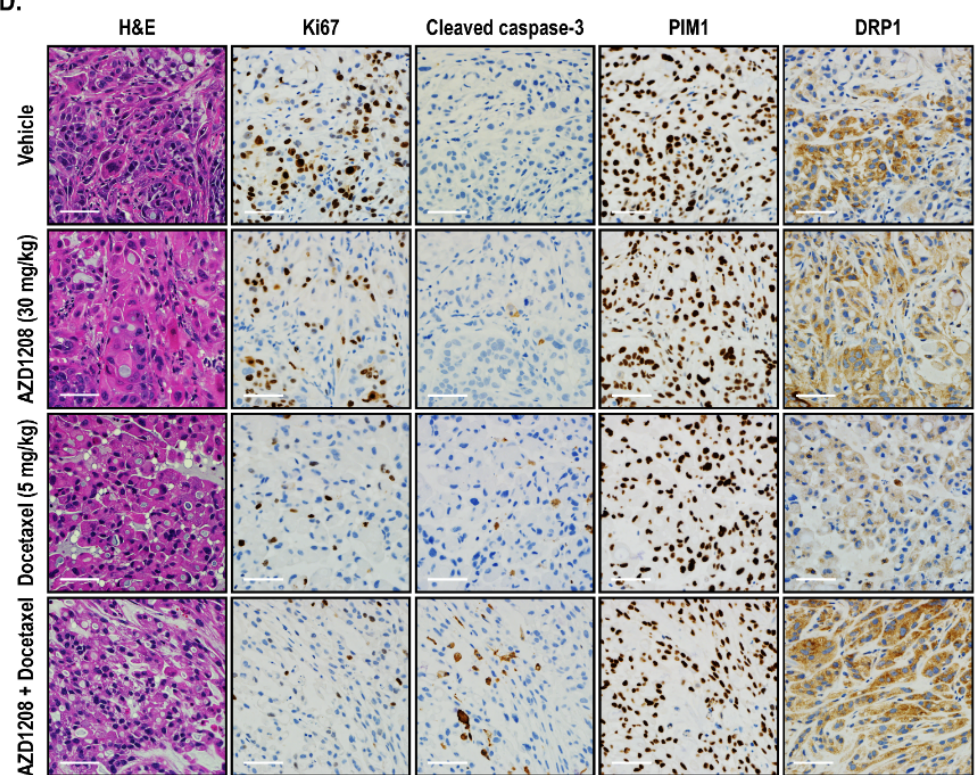

c.

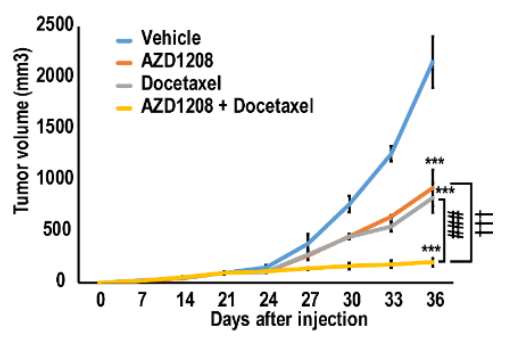

E.

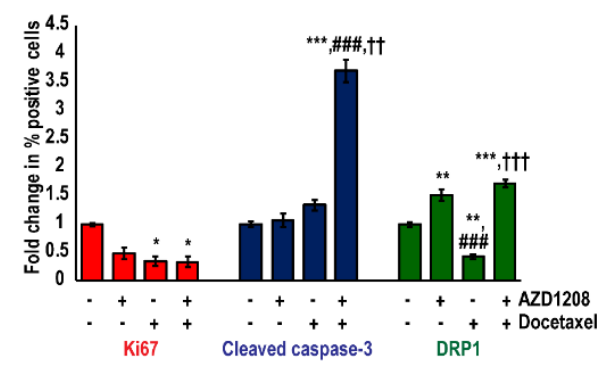

F.

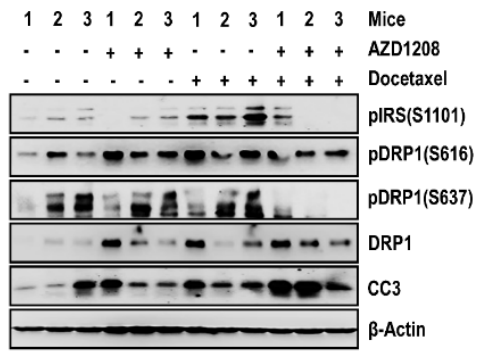

G.

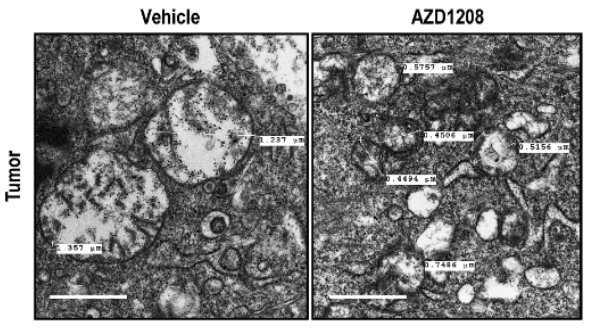

H.

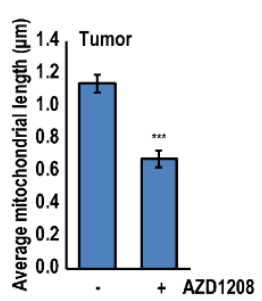

I.

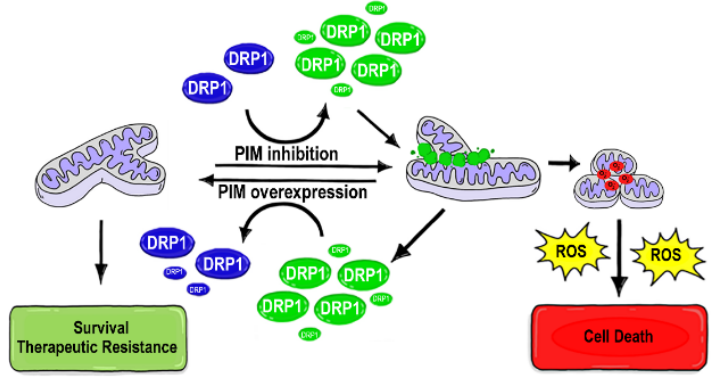


Figure S1: Live cell imaging showing increased MitoSOX staining (red) or superoxide production in $\mathrm{H} 1299$ cells treated with AZD1208 $(5 \mu \mathrm{M})$ for $2 \mathrm{~h}$. Scale bars, $100 \mu \mathrm{m}$.

Figure S2: (A) Representative immunofluorescence images showing RFP-PIM1 transfection in RKO (colon cancer) cells. Western blot confirming increased DRP1 phosphorylation at S637 upon PIM1 overexpression. (B) Indicated colon cancer cells stained for TOM20 showing decrease in fragmented mitochondria with PIM1 overexpression. (C) Prostate (DU145) and breast (SUM159 and MCF7) cancer cells showing increased mitochondrial fragmentation (TOM20 staining) and altered DRP1 levels and/or phosphorylation with PIM447 treatment. (D) Fold change in fragmented mitochondria in indicated cells treated with PIM447. Red arrowheads and mitochondria within red dotted line indicate fragmented mitochondria $(B \& C)$. Results are expressed as mean \pm SD of three independent experiments. ${ }^{* * *}, \mathrm{p}<0.001$ vs RKO; vs DMSO. Scale bars, $20 \mu \mathrm{m}$.

Figure S3: (A) TEM analysis showing increased mitochondrial fragmentation in H1299Keap $1^{-/-}$ cells treated with AZD1208 $(5 \mu \mathrm{M})$ for $24 \mathrm{~h}$. Scale bars, $2 \mu \mathrm{m}$. Histogram represents quantification of TEM images showing a significant change in the proportions of elongated (> $1.5 \mu \mathrm{m}$, yellow), intermediate $(1-1.5 \mu \mathrm{m}$, blue) and fragmented $(<1 \mu \mathrm{m}$, red) mitochondria. (B) Immunoblotting in H1299 and H1299Keap ${ }^{-/-}$cells treated with AZD1208 $(5 \mu \mathrm{M})$ for $0,24 \mathrm{~h}$ and 48h. Results are expressed as mean \pm SD of three independent experiments. ${ }^{*}, p<0.05$ vs DMSO.

Figure S4: (A) Immunofluorescence analysis of TOM20 in Drp1-knockdown lung cancer cells (A549shDRP1 and H460shDRP1). Magnification 60X; scale bars, $20 \mu \mathrm{m}$; red arrowheads indicate fragmented mitochondria $(<1 \mu \mathrm{m})$. Histogram representing fold change in the proportion of fragmented mitochondria. Results are expressed as mean \pm SD of three independent 
experiments. ${ }^{* *}, p<0.01$ vs parental line. (B) MitoSOX (red) staining in Drp1 knockout lung cancer cells treated with PIM447 $(3 \mu \mathrm{M})$ for $2 \mathrm{~h}$. Results are expressed as mean \pm SD of three independent experiments.

Figure S5: (A) Heat map representing dose-response, (B) combination index $(\mathrm{Cl})$ representing synergy, and $(\mathrm{C})$ table showing $\mathrm{Cl}$ values in $\mathrm{A} 549$ and $\mathrm{H} 460$ cells cotreated with a range of AZD1208 ( $\mu M)$ and docetaxel (nM) for 72 h. (D) Heat map representing doseresponse, $(\mathrm{E})$ combination index $(\mathrm{Cl})$ representing synergy, and $(\mathrm{F})$ table showing $\mathrm{Cl}$ values in A549shDRP1 and H460shDRP1 cells cotreated with a range of AZD1208 $(\mu M)$ and docetaxel (nM) for $72 \mathrm{~h}$. (G) JC-1 staining in lung cancer panel with $\mathrm{H}_{2} \mathrm{O}_{2}$ treatment for $24 \mathrm{~h}$. Magnification 40X; scale bars, $20 \mu \mathrm{m}$. Results are expressed as mean \pm SD of three independent experiments.

Figure S6: Immunoblot analysis of indicated cells treated with (A) $\mu$ M concentration of AZD1208, (B) PIM1 overexpression plasmid (hPIM1) and PIM inhibitor (PIM447, $3 \mu \mathrm{M})$, (C) PIM447 (3 $\mu \mathrm{M})$, docetaxel (3 nM), or the combination for $24 \mathrm{~h}$.

Figure S7: Crystal violet cell viability assay, heat maps representing cell viability, and drug $\mathrm{IC}_{50}$ quantification in (A) wild type RAS (NCl-H2228) and (B) EGFR mutated (NCl-H1975) lung cancer cells after $72 \mathrm{~h}$ docetaxel or cisplatin treatment, alone, and in combination with AZD1208. Decrease in docetaxel or cisplatin IC $_{50}$ values AZD1208 at $5 \mu \mathrm{M}$ concentration indicated cell sensitization. 


\section{Fig $\mathbf{1}$}

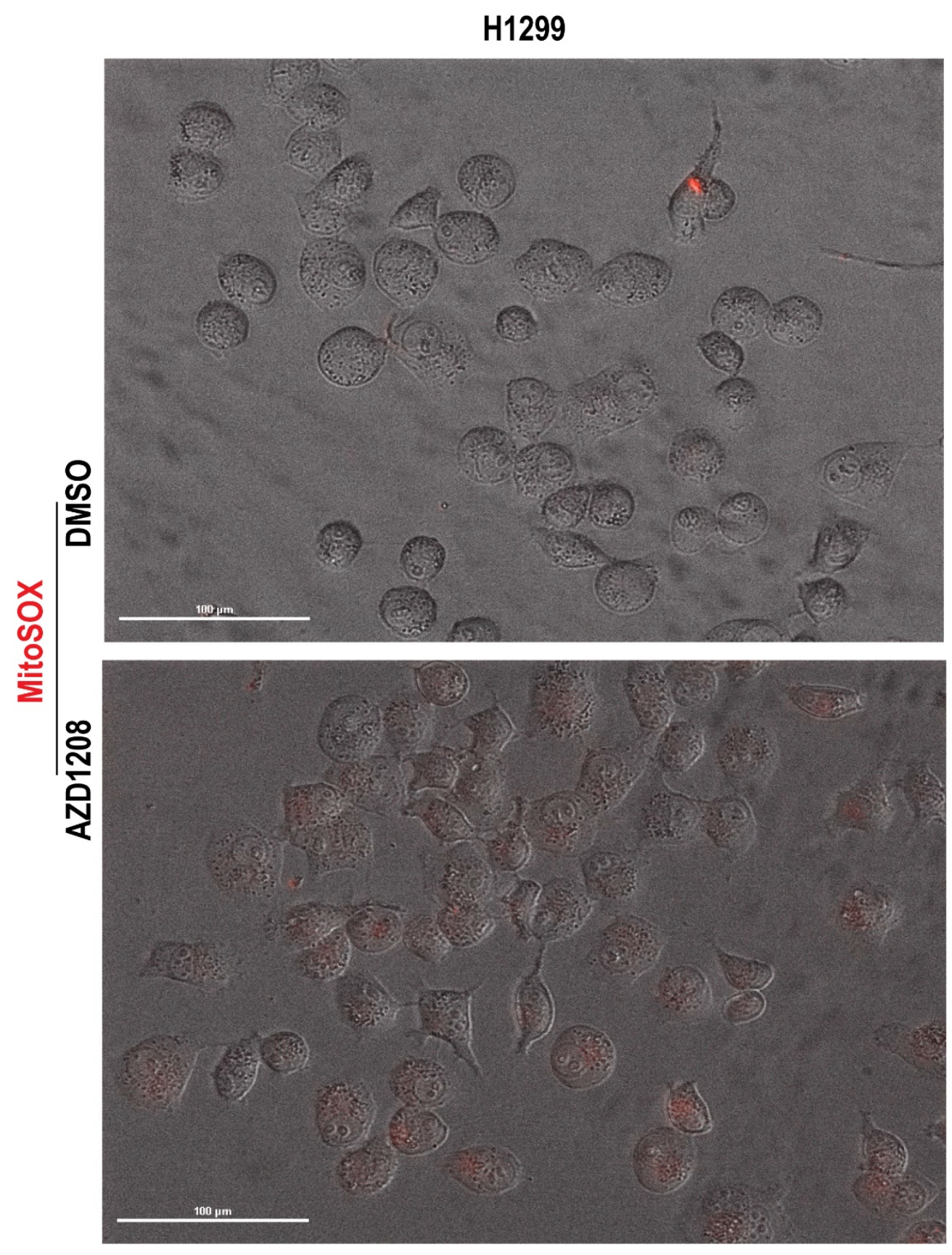


A.
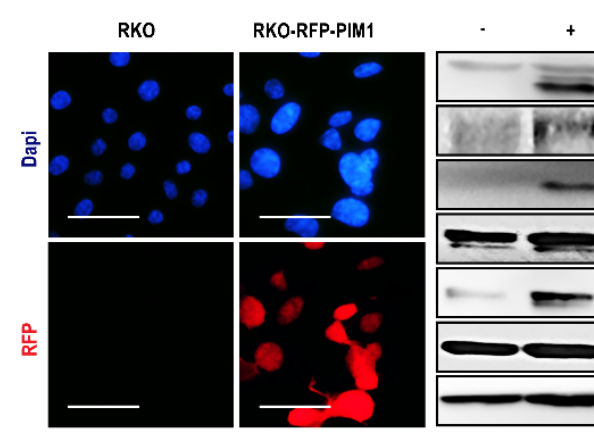

RFP-PIM1

PIM1

PIRS(S1101)

pBAD(S112)

pDRP1(S616)

pDRP1(S637)

DRP1

B-Actin
B.
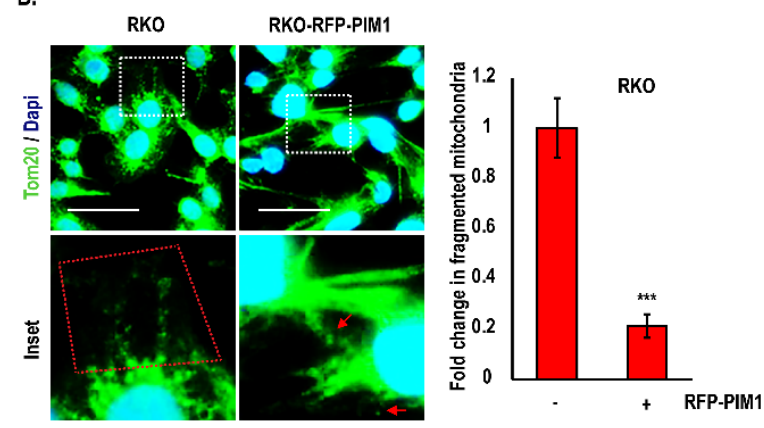

c.
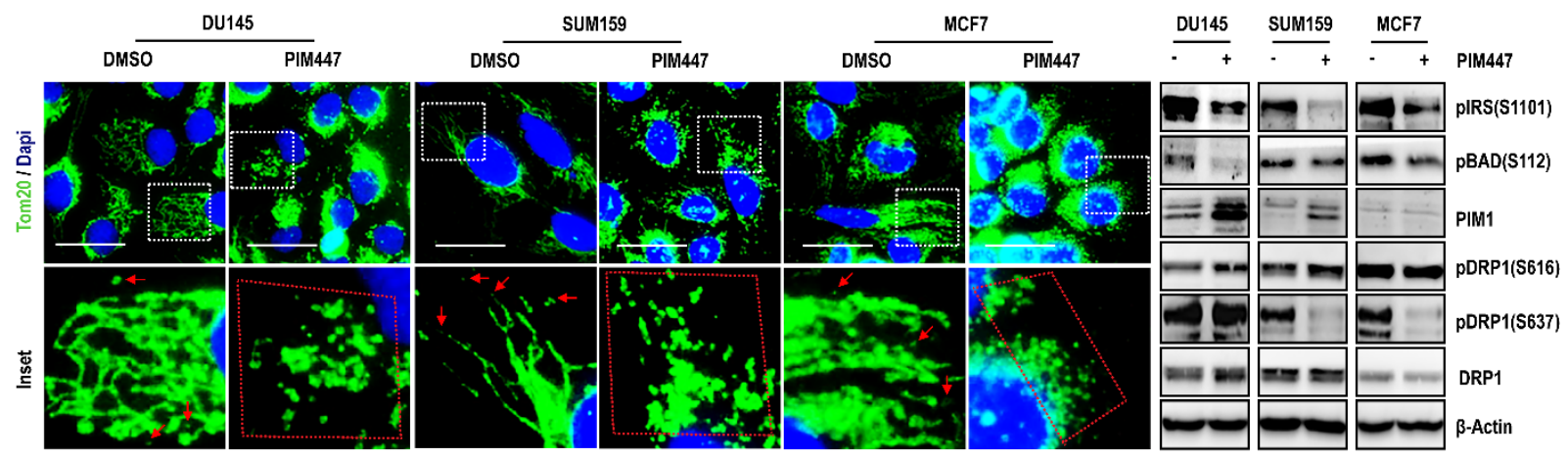

D.

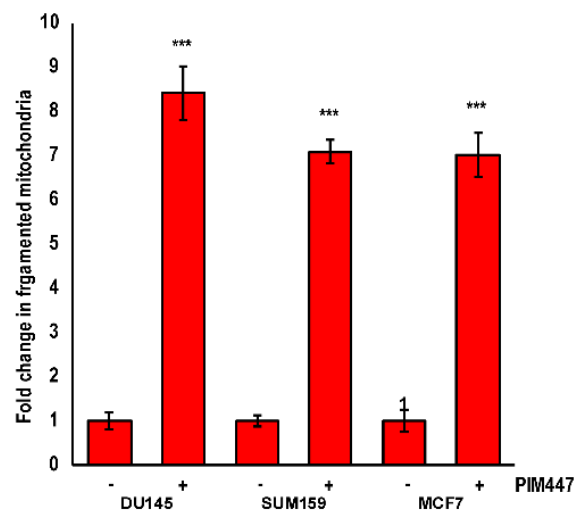


Fig S3

A.
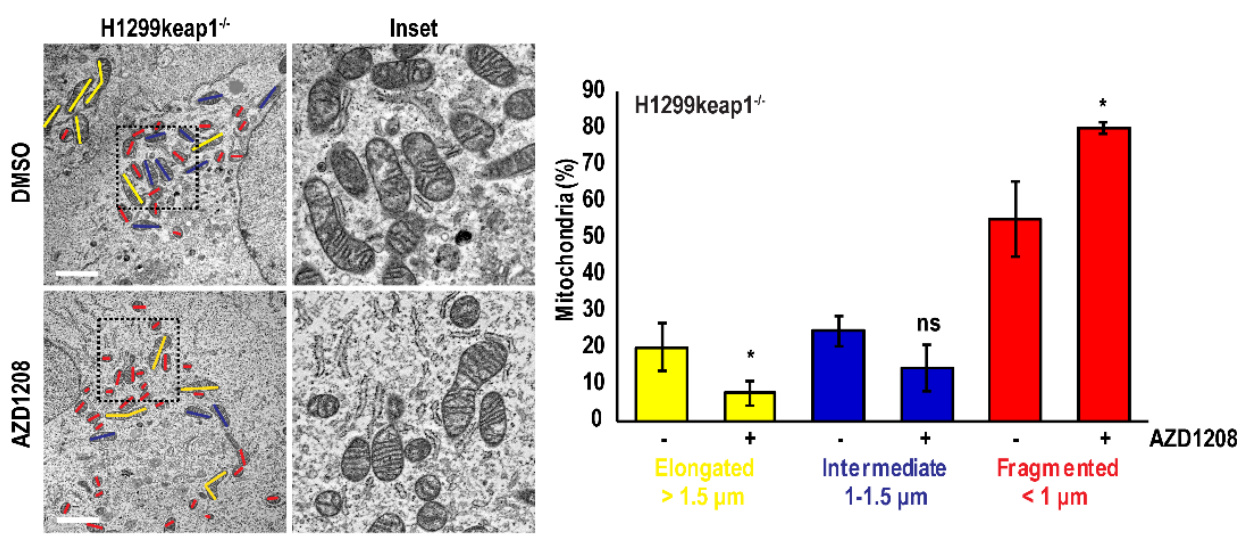

B.

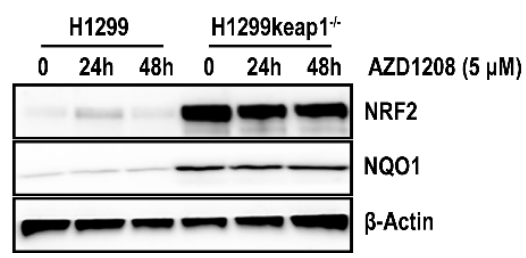


Fig S4

A.
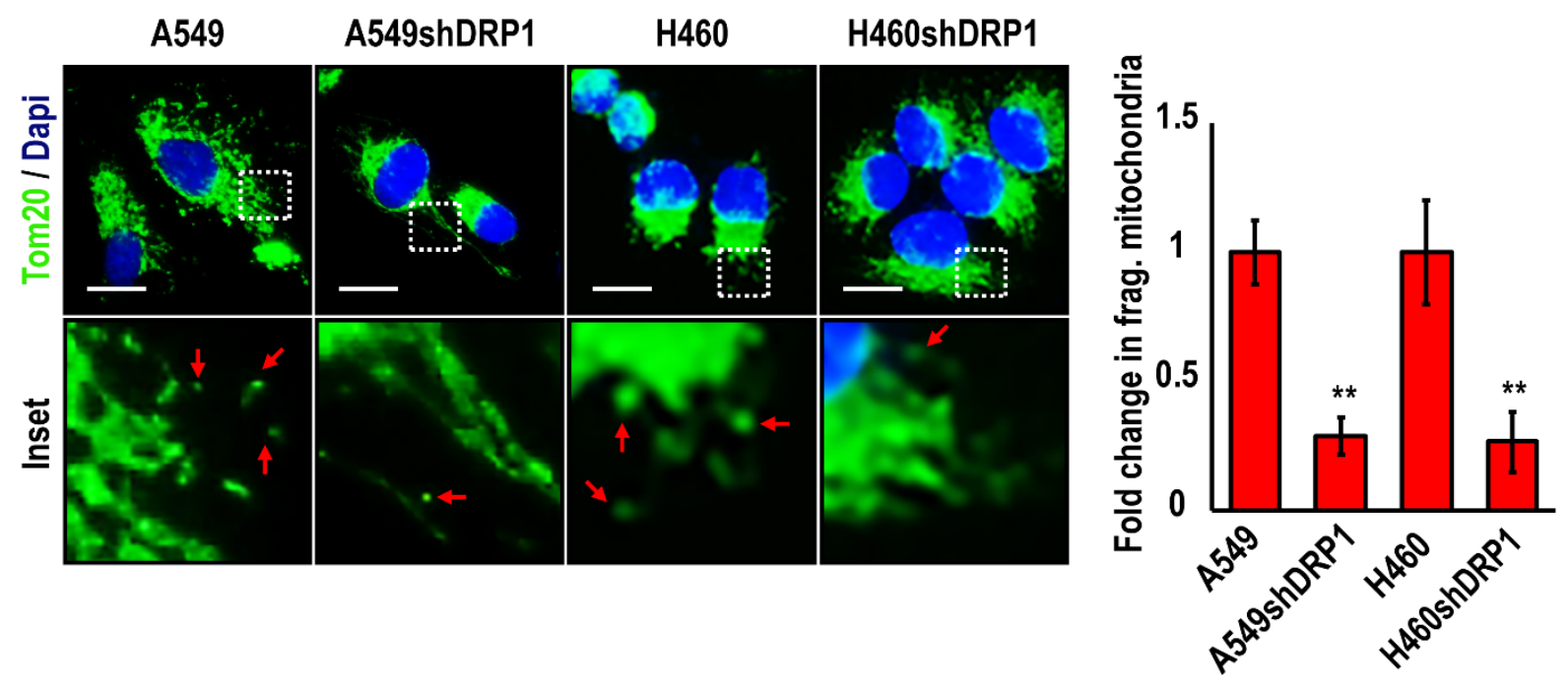

B.

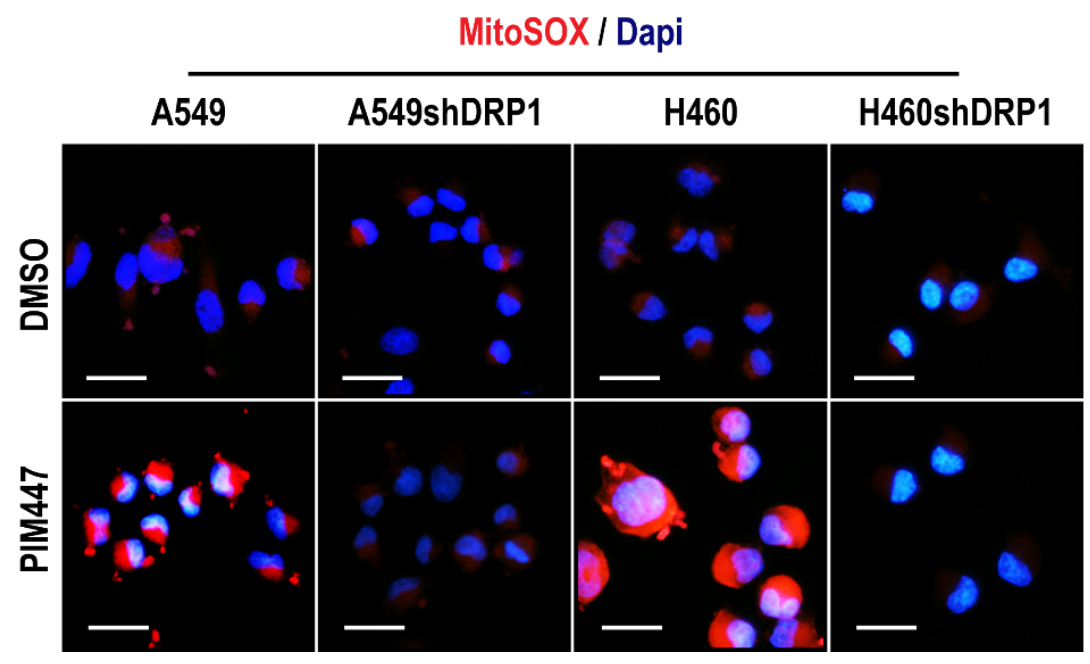


A.
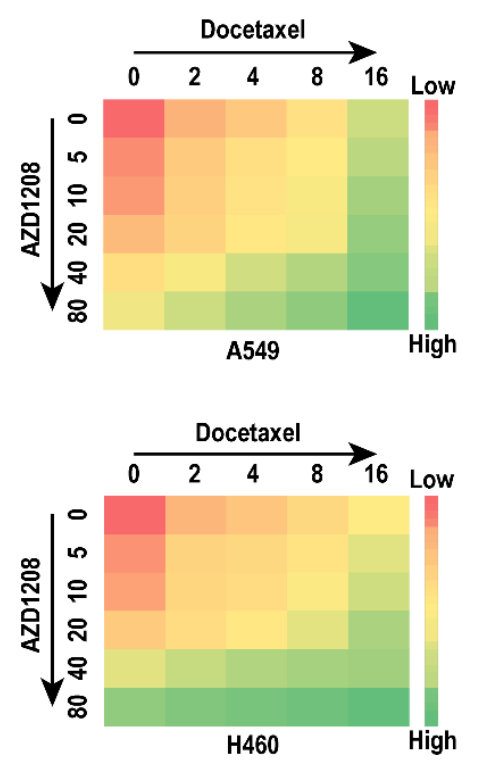

D.
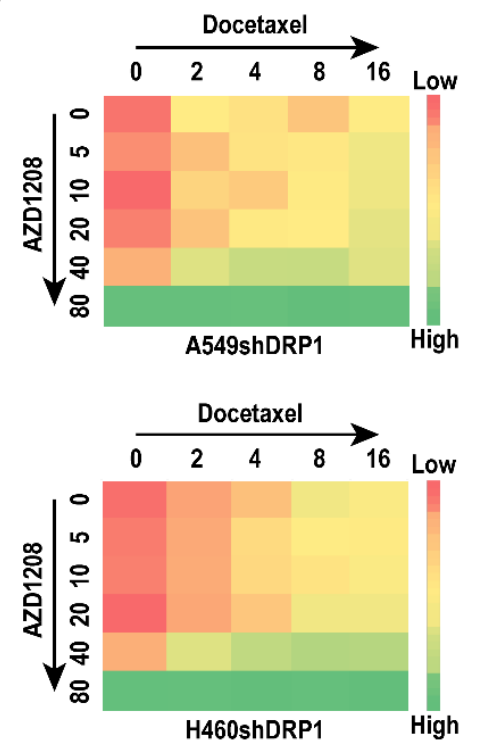

G.

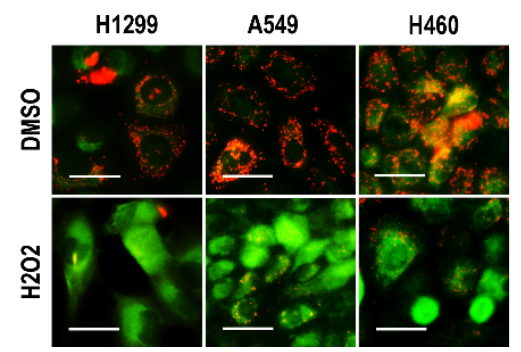

B.
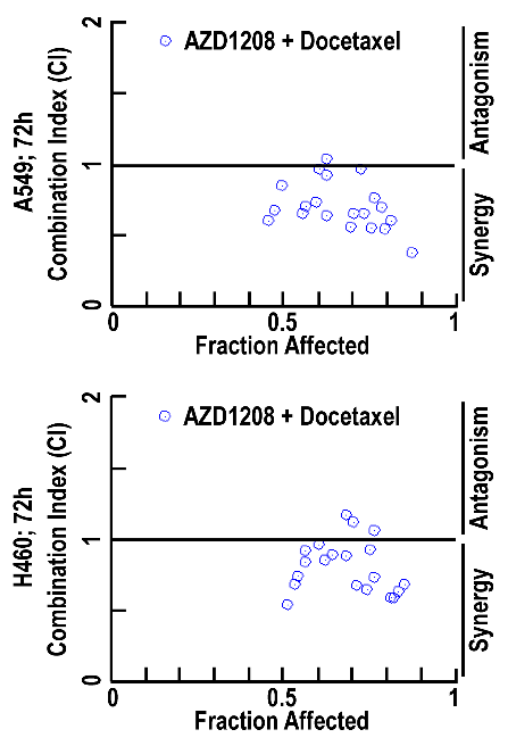

E.
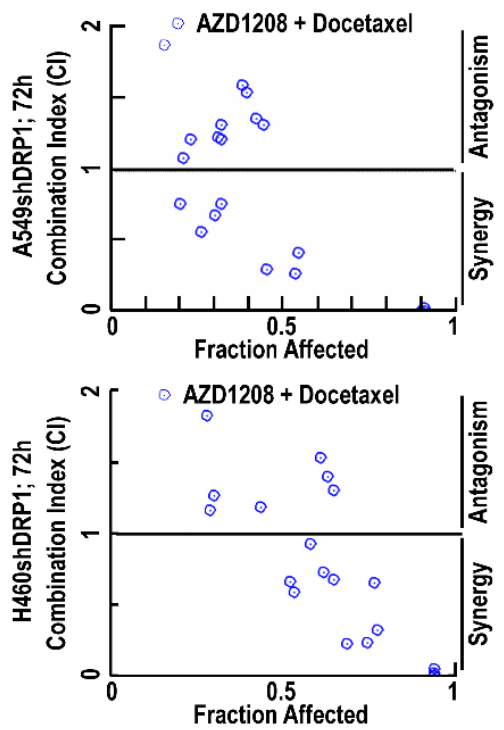

c.

Fig S5

\begin{tabular}{|c|c|c|c|}
\hline $0 s$ & Dose & 15 & \\
\hline $5 \mu \mathrm{M}$ & $2 \mathrm{nM}$ & 0.47 & 0.61 \\
\hline $5 \mu \mathrm{M}$ & $4 \mathrm{nM}$ & 0.57 & 0.67 \\
\hline $5 \mu \mathrm{M}$ & $8 \mathrm{nM}$ & 0.62 & 0.97 \\
\hline $5 \mu \mathrm{M}$ & $16 \mathrm{nM}$ & 0.74 & 0.98 \\
\hline $10 \mu \mathrm{M}$ & $2 \mathrm{nM}$ & 0.49 & 0.69 \\
\hline $10 \mu \mathrm{M}$ & $4 \mathrm{nM}$ & .58 & 0.71 \\
\hline $10 \mu \mathrm{M}$ & $\mathrm{nM}$ & 64 & 0.94 \\
\hline $10 \mu \mathrm{M}$ & $16 \mathrm{nM}$ & 0.78 & 0.77 \\
\hline $\begin{array}{l}\text { Dose } \\
\text { AZD1208 }\end{array}$ & $\begin{array}{c}\text { Dose } \\
\text { Docetaxel }\end{array}$ & $\underset{\text { Effect }}{\mathrm{H} 4}$ & ${ }^{50} \mathrm{Cl}$ \\
\hline $5 \mu \mathrm{M}$ & $2 \mathrm{nM}$ & 0.52 & 0.54 \\
\hline $5 \mu \mathrm{M}$ & $4 \mathrm{nM}$ & 0.55 & 0.74 \\
\hline $5 \mu \mathrm{M}$ & $8 \mathrm{nM}$ & 0.61 & 0.97 \\
\hline $5 \mu \mathrm{M}$ & $16 \mathrm{nM}$ & 0.69 & 1.18 \\
\hline $10 \mu \mathrm{M}$ & $2 \mathrm{nM}$ & 0.54 & 0.69 \\
\hline $10 \mu \mathrm{M}$ & $4 \mathrm{nM}$ & 0.57 & 0.84 \\
\hline $10 \mu \mathrm{M}$ & 81 & 0.65 & 0.89 \\
\hline $10 \mu$ & $\mathrm{nM}$ & 0.71 & 1.12 \\
\hline
\end{tabular}

F.

\begin{tabular}{|c|c|c|c|}
\hline \multirow{2}{*}{$\begin{array}{c}\text { Dose } \\
\text { AZD1208 }\end{array}$} & \multirow{2}{*}{$\begin{array}{c}\text { Dose } \\
\text { Docetaxel }\end{array}$} & \multicolumn{2}{|c|}{ A549shDRP1 } \\
\hline & & Effect & $\mathrm{Cl}$ \\
\hline $5 \mu \mathrm{M}$ & $2 \mathrm{nM}$ & 0.21 & 0.77 \\
\hline $5 \mu \mathrm{M}$ & $\mathrm{nM}$ & .31 & 0.69 \\
\hline $5 \mu \mathrm{M}$ & $8 \mathrm{nM}$ & 0.32 & 1.24 \\
\hline $5 \mu \mathrm{M}$ & $16 \mathrm{nM}$ & 0.39 & 1.60 \\
\hline $10 \mu \mathrm{M}$ & $2 \mathrm{nM}$ & 0.27 & 0.57 \\
\hline $10 \mu \mathrm{M}$ & $4 \mathrm{nM}$ & .24 & 1.22 \\
\hline $10 \mathrm{\mu M}$ & $8 \mathrm{nM}$ & .33 & 1.21 \\
\hline $10 \mu \mathrm{M}$ & $16 \mathrm{nM}$ & 0.40 & 1.55 \\
\hline $\begin{array}{c}\text { Dose } \\
\text { AZD1208 }\end{array}$ & $\begin{array}{c}\text { Dose } \\
\text { Docetaxel }\end{array}$ & $\begin{array}{l}\text { H460sh } \\
\text { Effect }\end{array}$ & $\begin{array}{c}\text { DRP1 } \\
\mathrm{Cl}\end{array}$ \\
\hline $5 \mu \mathrm{M}$ & $2 \mathrm{nM}$ & 0.29 & 1.61 \\
\hline $5 \mu \mathrm{M}$ & $4 \mathrm{nM}$ & .54 & 0.59 \\
\hline $5 \mu \mathrm{M}$ & $8 \mathrm{nM}$ & 63 & 0.74 \\
\hline $5 \mu \mathrm{M}$ & $16 \mathrm{nM}$ & 0.62 & 1.52 \\
\hline $10 \mu \mathrm{M}$ & $2 \mathrm{nM}$ & 0.30 & 1.26 \\
\hline & $4 r$ & 0.53 & 0.67 \\
\hline 10 & & & 0.9 \\
\hline $10 \mu \mathrm{N}$ & $16 \mathrm{nM}$ & 0.64 & 1.4 \\
\hline
\end{tabular}


bioRxiv preprint doi: https://doi.org/10.1101/863811; this version posted December 5,2019 . The copyright holder for this preprint (which was not certified by peer review) is the author/funder. All rights reserved. No reuse allowed without permission.

Fig S6

A.

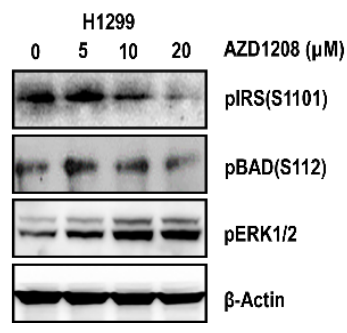

B.

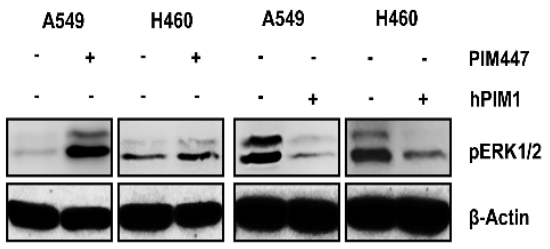

C.

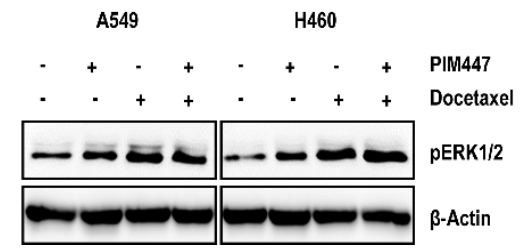


A.

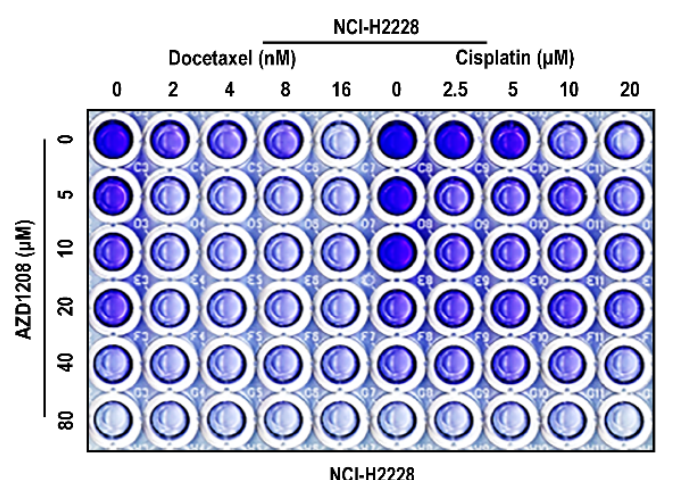

0

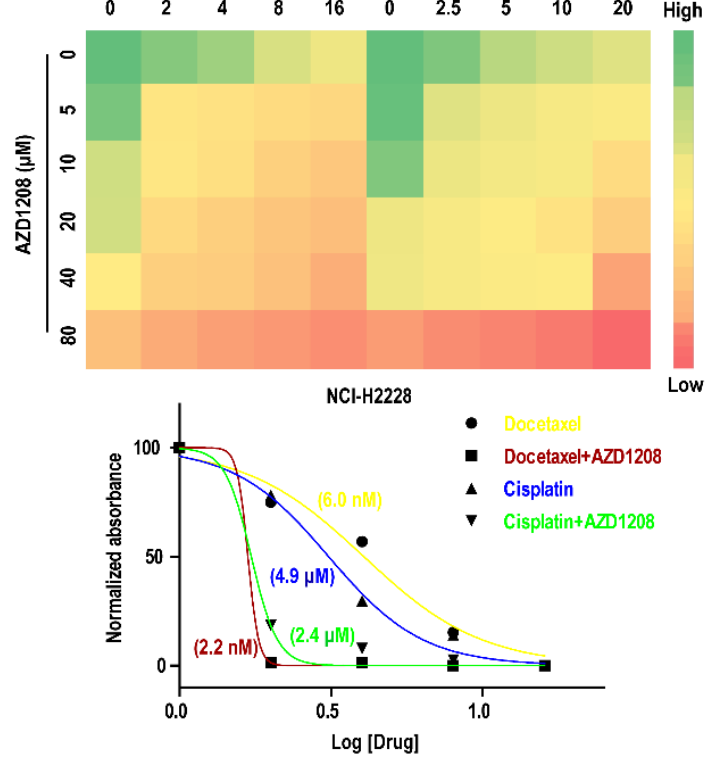

B.
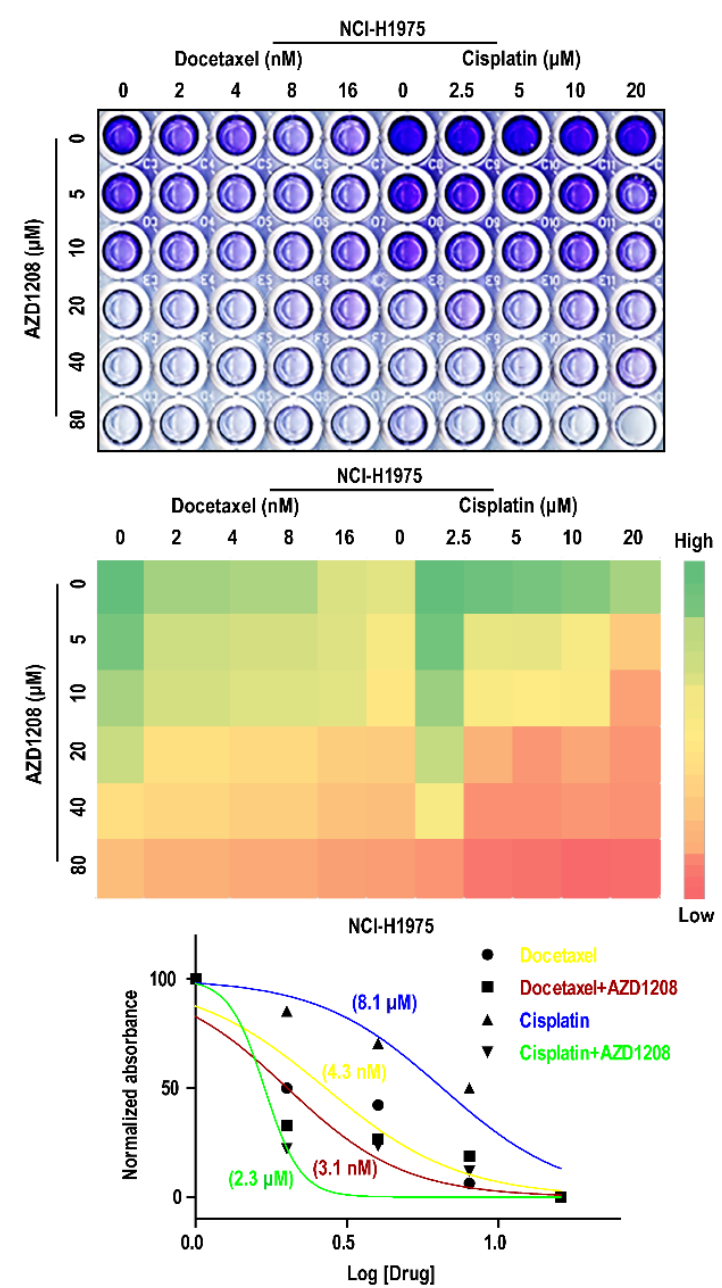